\title{
Auf dem Weg zur responsiven Demokratie? Online-Öffentlichkeitsbeteiligung in der Stadtentwicklung als aktiver Link zwischen Kommunalpolitik und Bevölkerung
}

\author{
Jörg Radtke • Sheree May Saßmannshausen
}

Online publiziert: 25. August 2020

(C) Der/die Autor(en) 2020

Zusammenfassung Öffentlichkeitsbeteiligung setzt mehr und mehr auf Online-Formate. Was können neue technische Möglichkeiten im Infrastrukturkontext leisten? Lassen sich so ein stärkerer Einbezug von BürgerInnen sowie eine bessere Vermittlung und Rückkopplung zwischen lokaler Bevölkerung, Politik und Fachplanung erreichen?

Die These des vorliegenden Beitrages besteht darin, dass, erstens, jenseits der klassischen Legitimationskonzepte Kategorien der Responsivität, Nähe und Sichtbarkeit für die Legitimitätsgenerierung entscheidend sind, welche, zweitens, durch Online-Formate befördert werden können. Anhand eines kommunalen Fallbeispiels werden einige Optionen für Echtzeit-Formate und Virtual Planning in der Stadtentwicklung exemplifiziert und aufgezeigt, wie Responsivität erzeugt werden kann. Hierbei kommen dem Design der Applikation, den Einstellungen von Politik und Verwaltung sowie einer passenden Beteiligungskultur/-strategie und umfassenden Mobilisierung der Bevölkerung, der gelingenden Verknüpfung mit Offline-Formaten, dem Vorhandensein von Ressourcen für den Betrieb und einem proaktiven Management der Beteiligungsplattform zentrale Bedeutung zu.

J. Radtke $(\bowtie)$

Fakultät 1, Seminar für Politikwissenschaft, Universität Siegen, Siegen, Deutschland

E-Mail: radtke@politikwissenschaft.uni-siegen.de

S. M. Saßmannshausen

Fakultät 3, Institut für Wirtschaftsinformatik, Universität Siegen, Siegen, Deutschland 


\title{
Making democracy respond: Online public participation as a means to reconcile municipal planning with local citizens' perspectives
}

\begin{abstract}
Public participation is increasingly focusing on online formats. What can new technical options in the context of infrastructure development accomplish, e.g. by employing interactive visualizations? Can greater involvement, better mediation and feedback between local population, politics and spatial planning be achieved?

In this contribution, we argue that apart from those derived from classical concepts on political decisions' legitimation, criteria such as responsiveness, proximity and tangibility appear crucial to generate legitimacy, which may be encouraged by online public participation offers. On the basis of an empirical case, we illustrate several modes of online-based real-time participation and virtual planning in municipal development and elaborate on how responsiveness can be created. The application's design and active management, attitudes towards participation held in the city's administration and council, their strategies (and resources) to mobilize the population to participate and to sensibly link the procedure and its outcomes to offline modes of participation as well as the local "participation culture" prove to be of decisive importance.
\end{abstract}

\section{Einleitung}

Im Forschungsprojekt Creactive Citizen wird eine experimentelle Online-Applikation für Öffentlichkeitsbeteiligung und Planungsvorhaben im Bereich der Stadtentwicklung erprobt. Dieser Beitrag präsentiert Ergebnisse der wissenschaftlichen Begleitung ihres Einsatzes. Grundsätzlich stellt digitale Kommunikation eine Chance dar, Verfügbarkeiten von Personen und Inhalten ortsunabhängig zu machen. Gleichzeitig werden Grenzen digitaler Zusammenkünfte offenbar: je mehr Personen in digitalen Räumen zusammenkommen, desto schwieriger werden die Bedingungen des Austauschs. Online-Formate können demnach insbesondere größere Veranstaltungen wie Bürgerversammlungen oder kollaborative Settings wie Arbeitsgruppen nicht ersetzen. Welche Rolle kommt also der E-Beteiligung zu? Eine naheliegende Funktion ist die Information, welche unilateral ausgerichtet (d.h. ohne direkte Möglichkeit der AdressatInnen, auf die Informationen zu reagieren) und internetbasiert organisiert sein kann. Rückmeldungen (Kommentar-/Forenfunktion) stellen eine nächste Stufe dar und implizieren bereits ein reaktives Moment. Daneben können Abstimmungen (Votings) zu Themen bzw. Vorschlägen erfolgen. Schließlich ist eine direkte Einbindung möglich - ob statisch per Eingabe oder durch die Gestaltung interaktiver Oberflächen, etwa mithilfe von Visualisierungen oder Mapping. Im Kontext der Öffentlichkeitsbeteiligung werden derzeit insbesondere Ideeneinbringung und Kommentierung, Abstimmungen und interaktives Mapping genutzt. Im Vergleich mit typischen Offline-Formaten der Öffentlichkeitbeteiligung - Bürgerversammlungen, Dialogveranstaltungen und Arbeitsgruppen - fällt auf, dass deren Besonderheiten gerade nicht durch Online-Angebote abgebildet werden. Daher wird Online-Beteiligungsformaten häufig eine Ergänzungsfunktion zugesprochen; zumal sie oftmals eine höhere Barrierefreiheit und Zugänglichkeit aufweisen, da sie mit Blick auf Raum 
und Zeit weniger gebunden sind. Die Aufgabe des Forschungsprojektes besteht darin, erweiterte kreative Optionen der digitalen Öffentlichkeitsbeteiligung auszuloten. Auf theoretischer Ebene wird im Kern die Frage adressiert, inwiefern E-Beteiligung die politische Legitimation öffentlicher Vorhaben erhöhen kann. Dazu werden insbesondere die drei Dimensionen politischer Legitimität (Input, Throughput, Output) betrachtet:

- Wie erhöht Online-Beteiligung politische Legitimität mit Blick auf Einbezug der Bevölkerung, Prozessgestaltung sowie Auswertung und Vermittlung von Ergebnissen?

- Inwiefern wird durch Online-Formate die Verständigung zwischen der Kommunalpolitik und der Bevölkerung verbessert?

- Kann über Online-Beteiligungstools eine stärkere Rückkopplung zwischen Input und Feedback der Bevölkerung und dem gremienbasierten Entscheidungsfindungsprozess erreicht werden?

Erweitert wird die Betrachtung um folgende Aspekte:

- Aktivierung und Mobilisierung (Wie können wenig beteiligungsaffine Personen besser erreicht werden? Wie können Jugendliche stärker angesprochen werden?),

- Attraktivität der Beteiligungsmöglichkeit (Wie kann Interesse erzeugt und innerhalb des Prozesses aufrechterhalten werden?) sowie

- Funktionen des Online-Tools (Wie lassen sich einschlägige Applikationen möglichst niedrigschwellig und gebrauchstauglich gestalten, während sie gleichzeitig die Komplexität der Thematik ausreichend abbilden?).

Hierbei untersuchen wir, inwieweit neue Wege der Legitimation politischer Entscheidungen durch andere Formen der Kommunikation, des Sich-Einbringens und der Äußerung von Feedback; kurzum: durch veränderte Modi der Responsivität erschlossen werden können. Im Folgenden werden zunächst Bezüge zu den theoretischen Ansätzen demokratischer Legitimität mit einem besonderen Fokus auf der Responsivität lokaler Politik hergestellt. Sodann wird der Forschungsstand zu bürgerschaftlicher Mobilisierung und Stadtplanung gespiegelt. Hieran schließen im vierten Kapitel Ausführungen zur Methodik und die Beschreibung des empirischen Anwendungsfalls sowie im fünften Teil die Darstellung der Analyseergebnisse sowie daraus abgeleitete Erkenntnisse hinsichtlich der Anforderungen an technische Beteiligungslösungen an. Im Fazit werden Schlussfolgerungen für den zukünftigen Einsatz von Online-Beteiligung in der Stadtplanung gezogen.

\section{Theoretische Bezugspunkte: Legitimitätsgenerierung und Bürgerbeteiligung}

\subsection{Konzepte demokratischer Legitimität}

Konzeptionen demokratischer Legitimität stützen sich häufig auf das Prozessmodell der Input-, Throughput- und Output-Dimensionen (Easton 1965). Inzwischen wird insbesondere die Bedeutung des Throughput hervorgehoben, ,,werden doch hier die 
in den politischen Prozess eingespeisten Interessen, Präferenzen, Werte, Normen und Ideen (vor allem) durch die politischen Akteure innerhalb des verfassungsrechtlichen Rahmens zu Politikergebnissen verarbeitet“ (Kneip und Merkel 2020, S. 31). Im Rahmen des Prozesses können Input-Präferenzen neutralisiert werden und Verzerrungen aufgrund illegitimer Interventionen durch private oder öffentliche Akteure auftreten (ebd.). In seinen Arbeiten zu demokratischer Legitimität fokussiert auch Rosanvallon (2011) stark den Throughput-Prozess: mit den Dimensionen Unparteilichkeit, Reflexivität und Nähe betont er Aspekte, die jenseits der formal-normierten Architekturen von Staat und Öffentlichkeit liegen. Das Konzept der Nähe meint dabei Ebenbürtigkeit von Staat und BürgerIn, Empathie, Offenheit und Transparenz. Der Staat soll BürgerInnen zuhören und ihre Belange und Ideen transparent in Entscheidungsprozesse einbinden. So wird Legitimität erhöht, da BürgerInnen sich ernst genommen und wertgeschätzt fühlen (Rosanvallon 2011, S. 172). Aus dieser Perspektive liegt eine Verknüpfung mit der pluralistischen Demokratie nahe, da hier die Abbildung gesellschaftlicher Pluralität und Meinungsvielfalt als wesentlicher Faktor demokratischer Legitimität gilt (Wintermantel 2020). Um diese stärker in politische Willensbildungsprozesse einzuspeisen und Legitimation zu erhöhen, wird insbesondere seit den 1960er und 1970er Jahren (,communicative turn“) auf Bürgerbeteiligung gesetzt. Nach wie vor ist das partizipative Paradigma ungebrochen, die Mehrheit der BürgerInnen wünscht sich mehr Mitsprachemöglichkeiten (Rademacher et al. 2020).

\subsection{Ungleichheitsbedingte Friktionen der Legitimität}

Seit einiger Zeit wird eine Krise politischer Repräsentation (Anter 2019) und des Parlamentarismus (Meinel 2019) sowie der liberalen Demokratie (Runciman 2020; Streeck 2013) in der Politikwissenschaft analysiert. Auch die demokratische Legitimität politischer Systeme wird im Zuge der Krisendiagnose als schwindend begriffen (Thiele 2019). Dies hat zum Aufstieg der Legitimitätspolitik geführt (Geis et al. 2012), wobei auch und gerade neue Kommunikationsformen die Legitimationsarchitektur nachhaltig verändert haben (Sarcinelli 2013).

Ein wesentliches Problem bei der Erreichung von Legitimität liegt in der Diskrepanz zwischen dem demokratischen Versprechen der Gleichheit (Wilson 2019) und der real existierenden Ungleichheit und Reproduktion derselben (Bude und Staab 2016; Butterwegge 2020; Rehbein et al. 2015). Gesellschaftliche Marginalisierung führt zu Wahlenthaltung und politischer Apathie, die daraus resultierenden Disparitäten der politischen Repräsentation begünstigen wiederum eine weitere Vertiefung der sozialen Gegensätze (Linden und Thaa 2014).

Für die additive Bürgerbeteiligung gilt: niedrigere Beteiligungsschwellen sind vorteilhaft und können die Legitimationsbasis erhöhen - allerdings liegt häufig strukturell begrenzte Partizipation vor, sodass die Formate selbst neue Legitimationsprobleme erzeugen (Träger 2020). Dennoch wird Bürgerbeteiligung theoretisch als wichtiges und wertvolles Surplus der repräsentativen Demokratie angesehen. Vergleichende Untersuchungen zeigen, dass Beratungsforen, Umfragen, Referenden und Instrumente partizipativer Politikgestaltung Policy-Wissen erhöhen, demokratisch-staatsbürgerliche Kompetenzen und öffentliches Engagement steigern sowie 
Entscheidungsfindungsprozesse unterstützen (Michels 2011). Responsivität könnte somit prinzipiell erhöht werden.

$\mathrm{Zu}$ bedenken ist aber: Die Effekte gelten nur für die im Regelfall wenigen TeilnehmerInnen, welche keinen repräsentativen Querschnitt der Bevölkerung abbilden (doppeltes Defizit) (ebd.; es beteiligen sich nach dem soziökonomischen Standarderklärungsmodell [SES] politischer Partizipation vornehmlich bildungs- und einkommensstarke Individuen, vgl. Brady et al. 1995; Schlozman und Brady 2018). Zahlreiche Untersuchungen zu Online-Beteiligung bestätigen diesen Befund, was in die Diagnose einer Digitalen Spaltung (Digital Divide) mündete (ungleich verteilte Ressourcen und Anforderungen bestimmen über Zugang und Nutzung - nicht alle nutzen Möglichkeiten zur Beteiligung gleichermaßen) (Marr und Zillien 2019; Rudolph 2019; Schlozman et al. 2010; Van Dijk 2006).

Bürgerbeteiligung ist somit individuell-selektiv vorteilhaft, nicht kollektiv-universell. Ein weiteres demokratisches Dilemma besteht darin, dass einerseits Viele involviert sein sollten, andererseits jedoch Politik im Leben der (designierten) Beteiligten oftmals nur eine untergeordnete Rolle spielt und ihr einschlägiges Wissen oft entsprechend gering ausfällt (Lupia und McCubbins 1998). Daher ist es sinnvoll, in konkreten Beteiligungsprozessen mithilfe von Methodenkombinationen Verbindungen zwischen spezifischen Kompetenzen und Motivationen aufzubauen (Steenbergen 2015). Eine typische Antwort besteht also darin, eine größere Vielfalt an innovativen und kreativen Verfahren und Input-Kanälen zu bieten und so gemäß dem pluralistischen Ideal den Input und die Bandbreite berücksichtigter Eingaben zu erhöhen (Merkel und Ritzi 2017, S. 247).

\subsection{Zur Responsivität von Bürgerbeteiligung}

Responsivität wird in der politischen Theorie im Sinne klassischer Verantwortlichkeit von EigentümerInnen und UrheberInnen (Pitkin 1967) sowie als Kongruenz der Einstellungen von RepräsentantInnen und WählerInnen verstanden (Eulau und Karps 1977). Hinsichtlich der Kongruenz unterscheiden Eulau und Karps unter Rückgriff auf das Miller-Stokes-Modell politischer Repräsentation (Miller und Stokes 1963) und Pitkin die vier Dimensionen Policy-, Service-, Verteilungs- und symbolische Responsivität. Während die symbolische Responsivität gegenwärtig in Postdemokratie-Debatten in Anschlag gebracht wird (Blühdorn 2013), hebt die empirische Forschung stärker die inhaltlichen, strukturellen und prozessualen Policy- und Verteilungs-Dimensionen hervor. So werden etwa Differenzen hinsichtlich der Definition guter Entscheidungen zwischen Bevölkerung und gewählten EntscheidungsträgerInnen analysiert (Giebler 2020).

Mit Blick auf Bürgerbeteiligung setzt Responsivität im Vorhof der Entscheidungsfindung an - nämlich bei der Kommunikation, bevor Sachdimensionen in konkreten Aushandlungsprozessen berührt werden. Auf Ebene der Verwaltungen besteht eine wesentliche Schwierigkeit in deren Neigung, sich der Öffentlichkeit zu entziehen (Szyszka 2020). Häufig läuft Online-Kommunikation in diesem Kontext eindimensional ab, indem Verwaltungen Input nach bestimmten Vorgaben aufnehmen und verarbeiten (Reddick et al. 2017). Responsivität wird so bestenfalls symbolisch erreicht. Eine aktuelle Untersuchung zu Mitgliedern von Gemeinderäten konnte zeigen, dass 
Beteiligungsverfahren grundsätzlich dazu beizutragen vermögen, RepräsentantInnen und Repräsentierte einander näherzubringen und damit die Responsivität zu erhöhen (Wilker 2019, S. 306). Indes kommt es durchaus zu Konflikten angesichts intransparenter Ziele von Beteiligungsverfahren, wenn etwa BürgerInnen versprochen wird, ,,auf kommunale Entscheidungen Einfluss nehmen“ und ,sich aktiv und direkt an städtischen Themen beteiligen“ zu können, während sich Ratsmitglieder doch vorbehalten, ,Ideen der Bürger im Zweifelsfalle zu ignorieren und bloß auf eine Legitimation ihrer repräsentativ gefassten Entscheidungen hoffen“ (ebd., S. 306f.). Mitunter befürchten KommunalpolitikerInnen einen Kontrollverlust; zudem sind sie kaum davon überzeugt, dass BürgerInnen ausreichend über Kommunalpolitik wissen - vielmehr hätten sie nur ihre eigenen Interessen im Blick (ebd., S. 255 f., 278). Schließlich betrifft die digitale Spaltung auch die Kommunalpolitik selbst: Inaktive PolitikerInnen werden von Online-Diskursen ausgeschlossen (ebd.). Responsivität bedeutet zunächst ausgeglichene, unvoreingenommene und transparente Kommunikation, an der beide Seiten vollumfänglich teilnehmen. Der Austausch zu konkreten Policy-, Service- und Verteilungsfragen müsste, um einen tatsächlichen Transfer von Präferenzen, Inhalten und Programmatiken zu erlauben, durch die Prozessgestaltung (i. S. d. Throughput-Legitimität) und durch geeignete Instrumente und Formate (wie Online-Beteiligungsfunktionen) (a) die Interessenallokation, (b) Anschlussfähigkeit und (c) Rückkopplung/Feedback aktiv befördern. Dynamiken der Austauschverhältnisse im lokalen Akteursnetzwerk sind bislang noch wenig erforscht - häufig sind jedoch bei Online-Beteiligungsverfahren bestimmte Akteure/Gruppen überrepräsentiert, was die Ungleichheitsproblematik nochmals untermauert (Sæbø et al. 2011).

\subsection{Die lokale Ebene: Nähe zu lokaler Politik und Verwaltung}

Demokratische Entscheidungen lassen sich besser im Rahmen überschaubarer lokaler Politik treffen (Jörke 2019). Bürgerbeteiligung auf kommunaler Ebene verbindet klassischerweise unmittelbare Betroffenheit mit niedrigen Hürden (van Ackern 2017; Klages 2013; Michels 2012). Anknüpfend an Rosanvallons Überlegungen zur Nähe zielt Bürgerbeteiligung im Kontext öffentlicher Vorhaben darauf ab, BürgerInnen und Staat besser zu verbinden Römmele und Schober (2013). Online-Beteiligung weist sui generis einen Bezug zur Kommunalpolitik auf, da unmittelbare Betroffenheit und Themen mit lokalem Bezug ein stärkeres Interesse der Bevölkerung hinsichtlich der Beteiligung an sowie dem Ausgang von Entscheidungsfindungsprozessen implizieren und eine Rückkopplung über die repräsentativen Formate der Kommunalpolitik hinaus weithin als sinnvoll erachtet wird (Alonso 2009; Kearns et al. 2002; Wesselmann 2002).

In den vergangenen Jahren hat sich ein zusehends responsiveres Politikmodell ausgebildet: die Vermittlung politischer Inhalte und Entscheidungsprozesse an die Bevölkerung über Informations-/Präsentations-, Dialog- und Diskursformate. Der Trend zum Local Open Government (Schmidthuber et al. 2017) hat Hoffnungen auf einen Umbruch zugunsten agiler Verwaltung und ihrer Prämissen Transparenz, Partizipation und Kollaboration erzeugt (Bartonitz et al. 2018; Hill et al. 2014; Rölle 2020). Die Idee erhöhter politischer Responsivität durch die Schaffung neuer Verbindungen zwischen BürgerInnen, lokaler Politik und Verwaltung spiegelt sich 
in der Diskussion um die zusätzliche Einführung von Online-Formaten wider (Eom et al. 2018; Mossberger et al. 2013; Zittel 2010). Online-Beteiligung kann prinzipiell Nähe und Vertrauen zu lokaler Politik und Verwaltung erzeugen, ist in ihrem Erfolg indes auch selbst vom allgemeinen Vertrauen in die Kommunalpolitik abhängig (Nam 2012) und benötigt politische und administrative Unterstützung (Macintosh et al. 2009; Reddick und Norris 2013). Aus Sicht von Politik und Verwaltung ruht eine wesentliche Hoffnung auf dem Outcome von Online-Beteiligung, indem Ergebnisse einer konkreten Verwendung zugeführt werden, weswegen etwa VotingFormate und Mapping-Tools beliebt sind (Feeney und Welch 2012).

\section{Online-Beteiligung: Stand der Forschung}

Forschung zu Online-Beteiligung fokussiert stark soziale Ungleichheit, die der Beteiligung zugrundeliegende Motivation sowie die Offenheit und Transparenz einschlägiger Angebote (Scherer und Wimmer 2011). Die spezifischen Barrieren von Online-Partizipation sind wiederum sehr vielfältig: Niederberger und Dreiack (2018) identifizieren 26 einschlägige Faktoren, die sich verschiedenen Kategorien zuordnen lassen ${ }^{1}$. Kersting führt die fehlende Erkennung von Missbrauch, geringes Erklärungspotential, Auswertungsprobleme sowie Manipulation, Clicktivism, Slacktivism, Echokammern, Filterblasen, Falschinformationen und Trolling an (Kersting 2019, S. 117). Häufig besteht ein Dilemma darin, gleichzeitig Kontinuität und hohe Beteiligtenzahlen zu erreichen (Flügge und Gerrits 2016). Vorteile können hinsichtlich einer Verbesserung des wahrgenommenen Nutzens und der Nutzerfreundlichkeit (Naranjo Zolotov et al. 2018, S. 362), der Schaffung multipler Beteiligungsoptionen zur Abdeckung unterschiedlicher Bedürfnisse (Große 2018, S. 7), der Vorbeugung anti-pluralistischer Tendenzen wie Populismus (Galston et al. 2018), dem Informationsgewinn und der Mobilisierung konstatiert werden (Kersting 2019; Norris 2012).

\subsection{Mobilisierung und hybride Partizipation}

Eine Meta-Studie über 60 Analysen konnte einige Schlüsselfaktoren ermitteln, die die Bereitschaft zur Nutzung von E-Partizipationsangeboten erhöhen. Hierzu zählen wahrgenommener Nutzen, positive Einstellung, soziale Einflüsse, Vertrauen und erwarteter Aufwand (Naranjo Zolotov et al. 2018, S. 362). Nach einer weiteren Untersuchung sind Zufriedenheit mit den Online-Applikationen, die Responsivität der

\footnotetext{
1 Die in der Forschung beschrieben Hauptprobleme: (1) Verfahren meist nicht bekannt (Franzke und Kleger 2009; Kubicek 2014; Rossmann und Shanahan 2012), (2) zu lang empfundene Zeitspanne (Aragonès und Sánchez-Pagés 2009; Auschner 2008; Franzke und Kleger 2009; Gilman 2016; Kim und Schachter 2013; McNulty 2015), (3) fehlende IT-Kenntnisse (Gilman 2016; Masser et al. 2013), (4) fehlende Ideen und mangelndes Interesse (Auschner 2008; Holtkamp 2008), (5) Relevanz der Beteiligung wird bezweifelt (Klages und Daramus 2007), (6) fehlendes Vertrauen in Anbieter/Staat (Garcia et al. 2005; Gilman 2016; Hordijk 2005; Kim und Schachter 2013), (7) Konkurrenz staatlicher und kommerzieller Angebote (Pollino und Modica 2013) sowie Kommerzialisierung und Beliebigkeit (Loomans 2015; Bizjak 2012), (8) Erforderlichkeit institutioneller Ressourcen (Hugger und Walber 2010; Marr und Zillien 2019; Rudolph 2019), (9) Datenschutzprobleme, Trolling und Manipulation (Zobel und Weiß 2018), (10) negative Effekte bei der Integration Sozialer Medien befürchtet (Alcaide Muñoz et al. 2018; Conroy und Vaughn 2018).
} 
(Kommunal)Politik, Entwicklungsfortschritte der Beteiligten im Prozess, wahrgenommener Einfluss auf Entscheidungsfindungsprozesse sowie Bewertung der Transparenz von Politik und Verwaltung entscheidend (Kim und Lee 2012). Offensichtlich lassen sich diese Faktoren zumindest in Teilen beeinflussen. Im Umkehrschluss bedeutet das: unterschiedliche Designs erzeugen unterschiedliche demokratische Effekte; die Eignung eines bestimmten Designs ist entscheidend von der Art der politischen Themenstellung abhängig (Michels und De Graaf 2017). Zudem bestimmt auch die Funktionalität von Beteiligungsinstrumenten stark über Art und Ausmaß der Nutzung (Zheng 2017).

In der Forschung werden weiterhin die Mobilisierungs-, Verstärkungs- und Normalisierungsthese beschrieben. Erstere geht davon aus, dass Beteiligung bei Personen initiiert werden kann, die bislang nicht politisch partizipiert haben. Die zweite These vermutet, dass Beteiligungsinteresse und -intensität von Personen, die schon vorher einschlägig involviert waren sowie offline aktiv sind, durch Online-Angebote erhöht werden (Hoffmann et al. 2019). Mitunter wird auch angenommen, dass beide Effekte wirken (Nam 2012). Vorherrschend ist indes das Paradigma der Normalisierungsthese. In einer Metaanalyse von 38 Studien wurde der Einfluss des Internets auf die politische und zivilgesellschaftliche Beteiligung untersucht (Boulianne 2009). Die Ergebnisse zeigen sich ambig; es wurden zwar keine negativen Effekte beobachtet, allerdings ließen sich auch keine klar positiven Resultate konstatieren. Somit könnten beteiligungstheoretische Annahmen zur motivationalen Grundlage von Partizipation offline wie online gleichermaßen Gültigkeit beanspruchen (vgl. weiterhin Gibson und Cantijoch 2013; Kubicek 2017; Oser et al. 2013; Robra-Bissantz et al. 2017). Es scheinen sich darüber hinaus verstärkt Ergebnisse zu sammeln, welche die Mobilisierungsthese stützen (Boulianne 2018; IFOK 2018), wonach Online-Angebote das Offline-Engagement verstärken können. Um eher passive Personengruppen (,Bystander“) zu erreichen, werden niedrigschwellige Interaktions- und Interventionsformen diskutiert (Jost et al. 2020).

Nach Untersuchungen von Kersting (2019, S. 115) nutzen Online-Beteiligungsangebote häufig ältere Personen, die auch offline aktiv sind; am ehesten können jedoch jüngere Menschen mobilisiert werden. Zweifelsohne bestehen erhebliche Unterschiede zwischen Face-to-Face-Kontakten und digitalen Formaten (Shu und Gu 2018). Nach Kersting (2019) zeigt Offline-Beteiligung deutliche Vorteile bei Deliberation und langfristiger Vernetzung, weshalb eine Kombination aus digitaler und klassischer Partizipation (Blended Participation) als Optimum gelten kann insbesondere, wenn innovative Online-Formate genutzt werden (ebd.).

Zusammenfassend zeigt die Forschung, dass bessergestellte Bevölkerungsteile stärker online (wie offline) partizipieren. Teilweise können jüngere Personen und eher benachteiligte Gruppen jedoch stärker mobilisiert werden. Spezifische technisch-anwendungsbezogene Kompetenzen markieren essentielle Voraussetzungen, die Diskursqualität stellt eine besondere Schwachstelle dar. Besonders kontextuell und situativ angepassten Formaten, welche gezielt auf Nutzergruppen eingehen, wird eine hohe Bedeutung beigemessen. Derlei Schlussfolgerungen finden sich u. a. bei Escher (2013; Escher und Rosar 2016), der in Zweifel zieht, dass sich die Legitimität aus Sicht von BürgerInnen, die grundsätzliche Beteiligungsdefizite wahrnehmen, durch Online-Formate erhöht - entscheidender seien hier Output und Impact kon- 
kreter politischer Entscheidungen (Escher und Rottinghaus 2019). Zu bedenken ist schließlich auch: die Art der Online-Beteiligung kann von Fall zu Fall variieren, die Beteiligung als Phänomen selbst wird online schwerer greifbar und lässt sich insgesamt nur schwer bewerten (Meckel et al. 2014, S. 56).

\subsection{Online-Beteiligung und die Mobilisierung Jugendlicher}

Da vielfach angenommen wird, dass sich durch Online-Beteiligung speziell jüngere Personen besser erreichen lassen, wird der Einsatz einschlägiger Instrumente in diesem Zusammenhang verstärkt analysiert und diskutiert (Abdu et al. 2017; Kersting 2016; Kersting und Hocks 2019; Macintosh et al. 2003; Moser 2010). Häufig sind Jugendliche im öffentlichen Diskurs und bei politischer Partizipation kaum präsent (Head 2011), weshalb die politische Bildung jüngere Personen verstärkt über das Internet adressiert. Auch in diesem Kontext gilt die Digital-Divide-Diagnose (Forrester und Matusitz 2010). Nach Vickery (2017) führt gerade die Fokussierung auf Gefahren der Internetnutzung dazu, dass die eigentliche Herausforderung in Form der Schaffung gerechter Online-Beteiligungsmöglichkeiten für marginalisierte Jugendliche übersehen wird. Aus demokratietheoretischer Perspektive ist es entscheidend, gestaltende Zugänge zur Demokratieentwicklung zu schaffen (Ertelt 2012) und Separierte gezielt online anzusprechen (Collin 2015). Hier steht wiederum die Kompetenzentwicklung im Mittelpunkt (Johanning 2009), wobei Demokratiekompetenzen (Eisel 2016) stark auf Gestaltungskompetenzen (de Haan 2008) ausgerichtet werden können, da online zahlreiche Interventionsmöglichkeiten zur Verfügung stehen. So wird der Einbezug Jugendlicher in die Lokalpolitik (Soßdorf 2016; Weiß 2018) und bei der Stadtplanung (Saridar Masri 2018) inzwischen stärker thematisiert, wobei die Verbesserung von Verbindungen zur politischen Sphäre (Dong und Ji 2018) sowie die Motivation, online zu partizipieren, im Vordergrund stehen (Vanslambrouck et al. 2018).

\subsection{Online-Öffentlichkeitsbeteiligung in der Stadtplanung}

Online-Beteiligung spielt vor allem bei Policy-Making-Prozessen eine Rolle (Macintosh 2004), wobei Planungsprozessen öffentlicher Vorhaben (z. B. Bauwerke, Infrastrukturen) eine besondere Bedeutung zukommt, da sie starke Auswirkungen auf das öffentliche Leben haben (Silva 2010). Die Kommunikation und Abstimmung mit der Bevölkerung spielt daher eine besondere Rolle (Brettschneider 2020). In der Planungstheorie und -forschung hat sich schon vor längerer Zeit das Ideal des kommunikativen, kooperativen und kollaborativen; kurzum: des beteiligungszentrierten Planens ausgeprägt (Booher 2018; Healey 2003, 2006; Innes und Booher 2003, 2010; Leffers 2019; Margerum 2002; Rotondo 2012; unter dem Stichwort Community Planning: Kelly 2010), das Vertrauen schaffen soll (Kumar und Paddison 2000). Ein besonderer Fokus liegt auf dem in der Öffentlichkeitsbeteiligung beliebten Themenfeld der Stadtentwicklung und Stadtplanung (Conroy und EvansCowley 2006; Damurski 2012; Donders et al. 2014; Eckardt 2016; Hennemann und Wiegandt 2010; Klammer et al. 2017; Kubicek 2010) sowie der Landschaftsplanung (Modica et al. 2013). Hierbei sollen nicht nur basale Informationsfunktionen erfüllt, 
sondern Inhalte kreativ erstellt und geteilt werden (Saad-Sulonen 2012). Seit einiger Zeit werden unter dem Schlagwort Civic Tech Online-Anwendungen diskutiert, die durch das Bereitstellen digitaler Informationen und Infrastrukturen einen offenen, partizipativen und kollaborativen Datenbearbeitungsprozess durch BürgerInnen ermöglichen (Baack et al. 2020; Bieber 2018; Gilman 2017; May und Ross 2018). Hinzu kommt die durch mobile Technologien und partizipative Sensoranwendungen ermöglichte Echtzeitpartizipation, die etwa bei über Crowdsourcing entstehenden Verkehrsstatistiken oder Lärmkarten Anwendung findet (Kanhere 2013). Hinter dem Ansatz der Online-Beteiligung im Stadtentwicklungskontext steht die Idee, „Stadt“ unter Einbezug zahlreicher Akteure über diverse Kanäle, transparent und mit hoher Diskussionsqualität gemeinsam zu entwickeln (Wiegandt et al. 2018). Kallus nähert sich einer zentralen Idee urbaner Partizipation über von Visualisierungstools, mithilfe derer ein gemeinschaftlicher Sinn für Orte sowie eine Stadtidentität ausgeprägt werden können (Kallus 2016, S. 1). Räume lassen sich auf diese Weise aus formellen Rahmungen der Stadtplanung herauslösen, stärker ins Alltagserleben der BewohnerInnen übertragen und so individuellen Ideen und Bedürfnissen zugänglich machen (ebd., S. 17). Der Fokus kann in diesem Kontext auf stärker deliberativen oder kreativen Ansätzen liegen (Charest et al. 2016), wobei gerade in diesem sehr plastischen Anwendungsfeld die Kreativität eine immer größere Rolle spielt. Diverse Beispiele von Stadtportalen bis zur Lärmaktionsplanung zeigen einen Trend zu virtuellen 3D-Modellen (Döllner et al. 2006; Jobst et al. 2010; Zhang und Moore 2014) und 3D-Realtime-Plattformen (Taube et al. 2020), die inzwischen ganze Städte abbilden (Schrotter und Hürzeler 2020). Die Effekte dieser Beteiligungsangebote können bislang nur grob abgeschätzt werden. Wie bei analogen Beteiligungsformen und anderen Online-Formaten kommt es zu Verlangsamungen beim Planungs- und Umsetzungsfortschritt (van Dijk 2006). Auch bleiben Differenzen zwischen FachplanerInnen und der Bevölkerung bestehen - etwa zwischen rationalen und emotionalen Motiven. Die Idee, solche eher grundlegenden Kontroversen durch OnlineBeteiligung abzumildern, indem eine stärker ganzheitliche Perspektive erschlossen wird, gilt als wenig aussichtsreich (Åström et al. 2011).

\section{Forschungsgegenstand und Vorgehen}

\subsection{Empirischer Anwendungsfall: Digitale Stadtentwicklung}

Die empirische Untersuchung bezieht sich auf einen konkreten Anwendungsfall in der Partnerkommune Olpe in Nordrhein-Westfalen. Ziel des Forschungsprojektes ist die Konzeptualisierung, Entwicklung und Evaluation eines Beteiligungstools für die lokale Öffentlichkeit. Hintergrund der Zusammenarbeit ist ein Beteiligungsprozess, den die Kommune im Zuge einer geplanten Neuentwicklung innerstädtischer Flächen initiiert hat. Den Ausgangspunkt bildeten Pläne der Stadtverwaltung für einen Neubau des Rathauses. Die politische Opposition im Gemeinderat (bestehend aus den Parteien SPD, Bündnis 90/Die Grünen, Freie Wähler sowie FDP) plädierte für eine Sanierung anstelle des Neubaus - primär aus Kostengründen. So kam es 2017 zu einem Bürgerentscheid, der mit einem deutlichen Votum für einen Rathausneu- 
bau ausging (63,5\% der abgegebenen Stimmen). Trotz der Bestätigung beschloss die Gemeinde die Initiierung eines Planungsprozesses für ein Integriertes Städtebauliches Entwicklungskonzept (ISEK) inklusive umfassender Öffentlichkeitsbeteiligung. Das Entwicklungskonzept bezieht sich auf den gesamten innerstädtischen Raum. Die Strategie des ISEK umfasste zwei zentrale Ansatzpunkte: eine Online-Beteiligungsmöglichkeit, wobei eine geobasierte Ideenkarte eingesetzt wurde (Vorschläge als individuelle Eintragungen auf der Stadtkarte), sowie (offline) Streifzüge durch die Innenstadt und spezialisierte Themenarbeitsgruppen. Zwecks Informierung über die Fortschritte wurden jährliche Bürgerveranstaltungen durchgeführt. Das Konzept für eine Online-Beteiligungsplattform sah zunächst interaktive, dreidimensionale Visualisierungsoptionen und den Einsatz von Augmented Reality (AR) vor; beim Transfer in eine für die Bevölkerung nutzbare Endversion offenbarten sich jedoch enorme technische Herausforderungen. Die Strategie wurde daher modifiziert. Einerseits werden innovative Visualisierungen mit kreativen Gestaltungsmöglichkeiten ohne Breiteneinsatz erprobt, andererseits soll ein Informations- und Kommentierungstool als mobile und browserbasierte Lösung eingesetzt werden. Damit werden die beiden Ebenen „Information und Diskussion von Ergebnissen und Vorschlägen“ (informativ-deliberative Beteiligungsvariante) sowie „Visualisierung und Ideeninput“ (visuell-kreative Beteiligungsvariante) unabhängig voneinander betrachtet und zusätzlich vergleichend untersucht.

\subsection{Methodik}

In diesem Beitrag werden Ergebnisse einer Vorstudie (mit entsprechend geringeren Fallzahlen) diskutiert. Dem Vorgehen liegt ein Forschungskonzept zugrunde, welches deduktiv und induktiv neben begründeten Vorannahmen und bekannten Studienergebnisse auch offen-explorative Erhebungen und entsprechende Verfeinerungen des Analyserahmens umfasst. Das Forschungsdesign entspricht demnach einem Mixed-Methods-Ansatz. Es wurde eine Kombination aus quantitativen Umfragen und qualitativen Interviews und Workshops eingesetzt, um komplementäre Ergebnisse sinnvoll zu verschränken. Die Daten wurden in den Jahren 2018 und 2019 erhoben.

Das durch die Forschungsfrage sowie den Forschungsstand grob vorgegebene Kategoriensystem setzt sich aus drei Kerndimensionen zusammen:

1. Persönliche Erfahrungen mit und Einstellungen zu Bürgerbeteiligung

2. Persönliche Erfahrungen mit und Einstellungen zu Online-Beteiligung

3. Anforderungen an das Design von Online-Beteiligungsapplikationen

Es bildete die Grundlage der Umfragen, für die Leitfragen der Experteninterviews sowie für die Themenblöcke der Workshops.

Die stärker theoriegeleiteten Kategorien der Legitimität (Input, Throughput, Output) sowie der Nähe und Responsivität (insbesondere die Kriterien Interessenallokation, Anschlussfähigkeit und Rückkopplung/Feedback) fanden vor allem bei dem Interviewleitfaden Berücksichtigung. Die Auswahl der InterviewpartnerInnen erfolgte entsprechend der konzeptionellen Ausrichtung auf den demokratischen Willensbildungsprozess: Zur Annäherung an die Dimensionen der Legitimität sowie an 
Responsivität und Nähe wurden KommunalpolitikerInnen aller im Gemeinderat der Partnerkommune vertretenen politischen Fraktionen $(n=7)$ und ein/e politische/r Mandatsträger/in interviewt. Zusätzlich wurden weitere, für den Stadtentwicklungsbeteiligungsprozess relevante Akteure aus Stadtverwaltung $(n=8)$ und der lokalen Zivilgesellschaft $(n=5)$ inkludiert. Der Fokus der Interviews lag auf Erfahrungen mit Bürgerbeteiligungsverfahren, insbesondere mit Online-Formaten. Zur Auswertung der Interviews wurden zunächst alle auf Beteiligungsverfahren bezogenen Aussagen paraphrasiert und kategorisiert. Offenes Codieren nach den Kriterien der Grounded Theory ermöglichte überdies die Genese weiterer relevanter Kategorien².

Zudem wurden zwei Online-Surveys durchgeführt, die sich stärker den unmittelbar anwendungsbezogenen Fragestellungen widmen. Die Items orientierten sich am oben erläuterten Kategoriensystem: erfragt wurden Einstellungen zur Bürgerbeteiligung (allgemein) und zur Online-Beteiligung, Beteiligungserfahrungen der Befragten und ihre Anforderungen an entsprechende Verfahren sowie insbesondere an Online-Tools. Da sie in Übereinstimmung mit dem Forschungsstand in den Interviews häufig Erwähnung fanden, wurde ein Fokus auf Jugendliche gelegt. So konnte eine Bevölkerungsgruppe, die in Beteiligungsprozessen unterrepräsentiert ist, aber gleichzeitig eine hohe Affinität zu Online-Medien aufweist, besondere Berücksichtigung finden. Die erste Umfrage richtete sich an die gesamte Stadtbevölkerung $(n=83)$, während die zweite speziell Jugendliche adressierte $(n=13$, Alter 14-19 Jahre, $62 \%$ weiblich, $38 \%$ männlich) und die Themen Freizeitverhalten, Engagement und Beteiligung, Beziehung zu PC, Internet und traditionellen Medien umfasste. Weiterhin wurden in Zusammenarbeit mit dem Jugendparlament der Kommune zwei Workshops veranstaltet, im Zuge derer thematisch fokussierte Gruppendiskussionen mit Jugendlichen stattfanden. Dabei wurden folgende Themenblöcke adressiert: Engagement und Beteiligung, Gestaltungsmöglichkeiten, Nutzung von (sozialen) Medien, Funktionen und Features von Apps sowie Wünsche für die Creactive Citizen App.

Schließlich wurden zahlreiche Eingaben von BürgerInnen ausgewertet, die im Rahmen von Informationsveranstaltungen, Streifzügen, Themenarbeitsgruppen sowie über die interaktive Webseite der Kommune zusammengetragen worden waren. Die primäre Zielstellung lag hierbei in der Identifikation bestehender Engagementstrukturen vor Ort als Anknüpfungspunkte für eine Mobilisierung für Online-Beteiligung. Die Bürger-Inputs wurden mittels einer thematischen Analyse ebenfalls kategorisiert. Auf dieser Grundlage ließen sich gemeinsame Bedürfnisse, Hemmnisse und Erfolgsfaktoren sowie Anforderungen an eine Beteiligungs-App feststellen.

\footnotetext{
2 So erweiterte sich der Kategorienkatalog (Bewertung/Motivation/Ziele/Erfolgsfaktoren von Bürgerbeteiligung und Onlinebeteiligung, Rahmenbedingungen und Kontextfaktoren, Teilnahmebereitschaft, demokratischer Prozess, Online-Offline-Verknüpfung, Funktionen des Onlinetools) insbesondere um die Dimensionen „Agenda-Setting“, „Bürgerorganisation“ und „Herausforderungen von Beteiligungsverfahren, Online-Beteiligung und E-Partizipationsplattformen“.
} 


\section{Empirische Ergebnisse}

Im Folgenden werden zentrale Ergebnisse zu den drei oben beschriebenen Hauptkategorien (Bürgerbeteiligung, Online-Beteiligung und Anforderungen an technische Lösungen) präsentiert und analysiert. Sie entstammen den Interviews, Umfragen, Workshops und Bürger-Inputs.

\subsection{Bürgerbeteiligung aus Sicht der Befragten}

Als Informationsquelle zu Beteiligungsmöglichkeiten kommt gemäß den Ergebnissen der Online-Surveys neben regionalen Zeitungen (73,5\%) auch Internet-Medien (64\%) eine zentrale Rolle zu - tatsächlich handelt es sich um zwei in ihrer Relevanz als nahezu gleichwertig bewertete bzw. in Kombination um die am häufigsten genutzte Kanäle. Die beliebtesten Bürgerbeteiligungsformen sind Abstimmungen und Meinungsabfragen über Bauprojekte, Bürgerversammlungen (69\%) und Informationsbzw. Dialogveranstaltungen (59\%) sowie Online-Befragungen und Abstimmungen (57\%). Online-Kommentierung (29\%) findet deutlich weniger Zustimmung. Zum Austausch über öffentliche Themen werden Online-Foren (19\%) und Soziale Medien $(31 \%)$ im Vergleich zu Offline-Räumen wie über Familie, Freunde, Bekannte (93\%), Nachbarschaft (58\%) oder Arbeit (46\%) weniger genutzt, obgleich Sozialen Medien ein ähnlich hoher Stellenwert wie Vereinen (31\%) zugeschrieben wird. Als besonders aufschlussreich erwiesen sich Angaben zu gewünschten Funktionen eines Online-Beteiligungstools: so wird vor allem eine Informationsfunktion betont; visuelle Darstellungen, Diskussions- und Vorschlagsfunktionen werden ebenfalls gewünscht. Online-Votings wird hier nur eine nachrangige Bedeutung zugeschrieben - ein Widerspruch zu den allgemeinen Beteiligungspräferenzen, der darauf schließen lässt, dass Abstimmungen implizit als Offline-Angebot gewertet werden. Die Präferenzabfragen zeigen, dass neben der obligatorischen Informierung auch Visualisierungs- und Diskursoptionen gewünscht werden, was eine stärkere Nutzung entsprechender Angebote erwarten lässt - wenngleich ein attitude-behavior gap berücksichtigt werden muss.

Im Allgemeinen unterstützen sämtliche befragten KommunalpolitikerInnen aller im Gemeinderat vertreten politischen Parteien Bürgerbeteiligung. Konkrete Erfahrungen waren aber nicht immer positiv:

wir hatten in der Rathaus-Diskussion, hatten wir eine Podiumsveranstaltung (...) Das war sicherlich ein Format, wo man gesagt hat, die da vorne erzählen was (...) Das war insgesamt häufig unbefriedigend für uns, da wir kein Meinungsbild hatten und für den Verlauf der Veranstaltung, ja die Bürger ein bisschen misstrauisch und dann sind noch zwei, drei, die lautstark sind, die vielleicht dann auch so eine Veranstaltung ins Unsachliche kippen. (Interview $03^{3}$ )

\footnotetext{
3 Die einzelnen Interviews wurden aufgrund der Anonymisierung der Interviewpartner nummeriert.
} 
Ähnlich beschreibt auch ein Verwaltungsmitarbeiter frontale, eher top-down geprägte Veranstaltungen, im Rahmen derer es zu Spannungen zwischen Verantwortlichen (,oben“) und dem Plenum (,unten“) kommen kann:

Ja, weil diese Frontal-Veranstaltungen einfach furchtbar sind. Man sitzt da vorne, 200 Leute sitzen einem gegenüber, haben „,so einen“ Kragen, und die Leute, die den meisten Kragen haben, kriegen das Mikrophon in die Hand, wo ich immer so denke: „Leute, ihr müsst mich hier nicht persönlich angreifen, das bringt so gar nichts". (Interview 04)

Indes wird von einem Paradigmenwechsel in der Kommune hin zu mehr Bürgerbeteiligung, Offenheit, Transparenz und Responsivität berichtet:

Im Moment werden neue Bürgerbeteiligungen erprobt, im Zusammenhang mit dem Thema Stadtentwicklung, Rathausneubau, Museumsneubau und so weiter.

Davor hatten wir weniger gute Erfahrungen (Interview 07)

Ein Kommunalpolitiker und Parteivorsitzender beschreibt eindrücklich den Vorteil unmittelbarer Rückmeldung durch die Bevölkerung für eine erhöhte Responsivität:

Für MICH; als Politiker, der unschätzbare Vorteil dieser Beteiligungen. Dass ich dadurch erfahre oder vermeintlich erfahre, was ist der Wille oder was ist die Meinung, die HERRSCHENDE Meinung in der Stadt. (Interview 11)

Doch zunächst muss nach Ansicht eines anderen Kommunalpolitikers ein Vertrauensverhältnis zwischen Politik, Verwaltung und der Bevölkerung aufgebaut werden. Es gelte, dem Eindruck von Scheinbeteiligung entgegenzuwirken, wobei die Bringschuld bei der Kommune läge:

Ich glaube auch, dass die Leute (...) auch eine gewisse Zeit brauchen, um das Vertrauen zu finden. Wollen die mich beteiligen oder sind das Showveranstaltungen? (...) Dass [das] dann endlich mal beim Bürger ankommt, so nach dem Motto: Mit dem, was ich hier vorschlage, ja, da passiert auch irgendwie mal was, ja? Und das ist jetzt nicht nur, damit die ihre Hausaufgaben für dieses Stadtentwicklungskonzept oder was halt machen, sondern es ist dafür da, weil sie sich wirklich für mich als Bürger interessieren. Und das gilt es zu beweisen. (Interview 13)

So wird Bürgerbeteiligung nach wie vor kritisch gesehen. Ein Parteimitglied fasst die Stimmung in der Bevölkerung zusammen und konstatiert ein verbreitetes Desinteresse an Mitwirkung explizit auch bei jüngeren Personen:

„Das hat doch gar keinen Zweck, dass man da sich beteiligt. Die machen doch, was sie wollen." Ja, so unter dem Schlagwort. (...) Die jungen Leute haben relativ großes Desinteresse an so Dingen teilzunehmen. (Interview 08)

Die Hürden der Bürgerbeteiligung werden durch Interviewte, die nicht in der Kommunalpolitik aktiv sind, ähnlich eingeschätzt. Ebenfalls wurde die fehlende Motivation der BürgerInnen, an partizipatorischen Verfahren teilzunehmen, angebracht. Damit die Befragten sich aktiv einbringen, müssten sie das Anliegen als 
dringlich und sie persönlich betreffend wahrnehmen. Die Antworten in den Surveys und der Interviewten zeigen, dass überwiegend globale Themen eine hohe Betroffenheit auslösen - beispielsweise „Klimawandel“ oder „Ungerechtigkeit“. Dennoch ist die Bereitschaft, sich zu beteiligen, wesentlich höher, wenn das Kriterium der Dringlichkeit erfüllt ist. Dieses Problem ist in der Forschung als Partizipationsparadoxon bekannt ${ }^{4}$. Die Gruppe der an Beteiligungsverfahren Teilnehmenden wird analog zum Stand der Beteiligungsforschung und der SES-These - als homogen eingeschätzt (,Ähnliche Leute, die sich beteiligen“, „Ältere, die Zeit haben“, ,,immer die gleichen, die da ihren Senf dazu tun“).

Beteiligungsformate wie Streifzüge durch die Stadt werden positiv beurteilt, doch fehlt es an Transparenz hinsichtlich des Outputs und Impacts der Veranstaltungen. Eine Kopplung der konkreten Umsetzung mit Feedback an Beteiligte wird von einem aktiven Bürger als sehr wichtig beschrieben:

Also die Spaziergänge fand ich eben auch gut. (...) bis auf die Transparenz, habe ich schon gesagt. Also man erfährt hinterher nicht, was daraus geworden ist (...). (Interview 01)

\subsection{Online-Beteiligung aus Sicht der Befragten}

Wie weitere Befragungen zeigten, nutzen viele BürgerInnen neben der Lokalzeitung (und deren Online-Präsenz) Facebook, um sich über aktuelle Geschehnisse in ihrer Stadt zu informieren. Die Kommunikation zwischen BürgerInnen und den Behörden erfolgt meist durch face-to-face-Kontakte oder in schriftlicher Form. Social Media wird bislang kaum genutzt, obwohl darin ,gerade für die jüngeren Zielgruppen“ Potenziale gesehen werden.

Die Befragten aus der Kommunalpolitik stehen Online-Beteiligung insgesamt positiv gegenüber. Gleichzeitig äußerten sie durchaus Bedenken - etwa hinsichtlich Manipulationsmöglichkeiten, Datensicherheit, Trolling und Hate Speech - sowie eine teils grundlegende Skepsis gegenüber Online-Angeboten. Der Bürgermeister sieht Chancen insbesondere für Personen, die bei größeren Plenarveranstaltungen passiv agieren, da online niedrigere Hemmschwellen bestünden. Der Einsatz einer interaktiven geobasierten Ideenkarte habe dies bereits zeigen können:

Diejenigen, die das (...) für nicht realisierbar halten in so einer großen Runde, die melden sich im Zweifelsfalle nicht. (...) Und da bieten sich eben durch so eine Plattform andere Möglichkeiten nochmal. (Interview 09)

Es muss niedrigschwellig sein. Und es hat ja unter anderem (...) diese interaktive Karte gezeigt, wenn das einfach zu bedienen ist, intuitiv zu bedienen ist, dann wird das auch angenommen. (Interview 09)

\footnotetext{
$4 \mathrm{Zu}$ Beginn der Planung eines Projektes können Änderungsvorschläge am ehesten berücksichtigt werden. Allerdings ist es in dieser frühen Planungsphase schwierig, Personen für die Beteiligung zu aktivieren. Während die Planung voranschreitet, schwinden Möglichkeiten der Einflussnahme, während das Interesse daran zunimmt (Dolde 2013; Alcántara et al. 2014, S. 124).
} 
Die Befragten ohne kommunalpolitischen Hintergrund sehen einen Vorteil der Online-Beteiligung darin, dass sich jüngere BürgerInnen besser erreichen lassen. In den Bürger-Inputs, die im Zuge des ISEKs erhoben wurden, wurde mehrmals die Bedeutung der Einbeziehung jüngerer Generationen unterstrichen. Tatsächlich fühlen sich Jugendliche nicht ausreichend berücksichtigt und ernst genommen. Sie befinden, die Politik interessiere sich nur „,manchmal“ für ihre Meinungen:

Es ist schwierig in der Masse ernst genommen zu werden. Schließlich ist der Großteil der Politiker mittleres bis Rentenalter. Hier wird man gerade als junger Mensch oftmals belächelt, da wir angeblich nicht so viele Erfahrungen haben, zu jung sind, nichts von den Themen verstehen oder angeblich nur nicht umsetzbare Ideen haben. Dies sehe ich anders, schließlich ist es wichtig, dass man in der Politik Meinungen aus allen Generationen und Alterstufen vertritt, da diese unterschiedliche Wünsche und Ideen haben. (Interview 90)

Hier lässt sich eine Diskrepanz zwischen Problemwahrnehmungen durch Erwachsene (hier: PolitikerInnen) und Jugendliche konstatieren. Dies wird von den befragten Mitgliedern des Jugendparlaments auch auf die Art der Kommunikation zurückgeführt, wobei digitale Remote-Kommunikation der konventionellen Face-toFace-Kommunikation gegenübersteht. Die beiden Kommunikationsformen werden als voneinander stark divergierend beschrieben:

Und dann kann man sich da manchmal nicht so genau austauschen, wie wenn man jetzt von Person zu Person miteinander reden würde, weil dann sagt man vielleicht auch Sachen, die man nicht sagen würde, wenn jemand vor einem steht. Oder man drückt sich anders aus. Und das finde ich halt schade, wenn das verloren geht. (Interview 31)

Die mehrheitlich politisch interessierten Jugendlichen nutzen vor allem ihre Smartphones, um sich zu informieren. Dabei greifen sie auf YouTube, verschiedene Nachrichten-Apps und Social Media zurück. Sie sind der Ansicht, dass Meinungen online gut ausgetauscht werden können, während sich echte Diskussionen schlechter webbasiert führen lassen:

Man sieht keine Emotionen. Keine Reaktionen. (...) Man bekommt natürlich ne Antwort, aber man sieht nicht genau, wie der Andere darauf reagiert. Also ob ich dir jetzt gegenübersitze und dir in die Augen schaue und sehe, wie sich dein Gesicht bewegt, ist was anderes, als wenn du mir ein Emoji schickst. (Interview 33)

Folglich braucht es zur stärkeren Inkludierung von Jugendlichen in Beteiligungsverfahren nicht nur digitale, sondern auch klassische Formate. Die von Seiten der kommunalpolitisch involvierten Befragten geäußerten Vorstellungen zur Verbindung von Online- und Offline-Beteiligung decken sich mit diesem Ergebnis und dem Forschungsstand. Offline-Formate sollen demnach vor allem den Einbezug digital inaktiver Bevölkerungsgruppen ermöglichen, wie ein älterer Parteivorsitzender argumentiert: 
es gibt Leute, die nehmen an dieser Informationswelt nicht teil. Die wollen aber auch informiert werden und wählen auch. So und deswegen ist es wichtig, dass man sich (...) auf die, die alten Informationsveranstaltungen dann besinnt. (Interview 06)

Die befragten VertreterInnen der Zivilgesellschaft nannten Anerkennung, Respekt und genuines Interesse an den Positionen der BürgerInnen als wesentliche Voraussetzungen für erfolgreiche Online-Beteiligungsverfahren. Ein Kommunalpolitiker spricht sich für eine erste entpolitisierte Phase in Beteiligungsprozessen aus, die durch eine sich zurücknehmende, passive Kommunalpolitik gekennzeichnet ist (,citizen first“). So sollen politische Einflüsse auf den Meinungsfindungsprozess reduziert werden:

Dass man eine Mehrheitsmeinung findet. Das könnte man natürlich nur schaffen, indem man schon diese Dinge argumentativ steuert. Und das ist aber, finde ich, NICHT Sinn der Sache. Weil, was jetzt endlich dann daraus GEMACHT wird, (...) entscheiden zum ersten Fachleute. Die sagen (...) das ist eine Sache, die ist eigentlich nicht machbar. Dann scheidet so etwas schon aus. Auch, wenn da 50 Leute hinter stehen. Aber, wenn man argumentativ führt, dann (...) hat man eine gewisse Art der Manipulation. Also wir haben uns auferlegt (...) Die Politik soll sich aus der Sache erstmal raushalten. (Interview 11)

Einerseits besteht in dieser Perspektive Ergebnisoffenheit, andererseits haben ExpertInnen das letzte Wort - das kann zu Verdruss bei Beteiligten führen. Uns interessieren in diesem Zusammenhang besonders die unterschiedlichen Perspektiven von Politik, Verwaltung und Bevölkerung, da (Online)Beteiligung die Verständigung zwischen den Sphären verbessern kann (Eom et al. 2018; Gauld et al. 2016; Zittel 2010). Die Chance der stärkeren Abbildung von Multiperspektivität wird auch in den Interviews beschrieben, allerdings mit Blick auf die obligatorische Konsensfindung sogleich problematisiert:

finde ich schon gut, weil vielleicht, sagen wir mal, die Leute, die es entscheiden, die sehen es aus einem Blickwinkel und die Leute, die in der Stadt leben, sehen es vielleicht es einem anderen Blickwinkel. Das ist vielleicht nicht außer Acht zu lassen. Die ganzen städtebaulichen Maßnahmen, die da hier gerade in Olpe passieren, viele regen sich darüber auf (...). Ist auch immer die Frage, wenn alle möglichen beteiligt werden, 1000 Leute, 1000 Meinungen, ob man da nachher zum Ende kommt, weiß ich auch nicht. (Interview 12)

Hier klingt ein kritisches Moment an: der Übergang von einer offenen Agenda Setting-Phase über die stärker konkretisierte Konzeption zum endgültigen Beschluss in der Politikformulierung. Arten und Umfänge der Bürger-Inputs, die dabei zulässig sind bzw. Berücksichtigung finden (können), verringern sich zwangsläufig sukzessive (was in der Beteiligungsforschung mithilfe einer Trichter-Metapher [funnel of causality, Campbell et al. 1960] umschrieben wird [Muncke 1972, S. 234] sowie in der Technikfolgenforschung als Collingridge-Dilemma bekannt ist [Collingridge 1980]). Die Befragten markieren denn auch den Punkt des Umschwungs von der 
offenen Planung hin zur konkreten Formulierung und Implementation als kritischen Moment:

Der zweite Punkt ist - wo ich mir wünschen würde, dass sich sowas auch wirklich kontinuierlich umsetzen lässt mit den Menschen, und wo ich die Gefahr eigentlich drin sehe - ist, dass wir jetzt momentan Beteiligungsprozesse haben, wo viele erstmal nur Wünsche äußern. Und irgendwann wird es natürlich auch zu einem Verfahrensschritt kommen, wo man bestimmte Dinge annimmt und umsetzt. Und bei anderen Dingen auch sagt, es ist nicht umsetzbar. Und dann fingen natürlich die kritischen Dinge auch an. (Interview 09)

Die geben uns das Problem und sind dann womöglich enttäuscht, wenn wir es nicht machen. Also Verwaltung ist dann traditionell immer schuld. Weil wir womöglich einen Grund haben, das nicht zu tun. (Interview 09)

Damit werden gleichermaßen ein kritischer Aspekt der pluralistischen Demokratie (Anspruch der Abbildung zahlreicher heterogener Perspektiven, Interessen und Präferenzen, die im Zuge der Umsetzung mitunter verloren gehen) sowie das demokratische Dilemma zwischen Partizipation und Systemeffektivität, die bei breiter Inklusion und Deliberation pluralistischer Perspektiven verringert würde, tangiert (Dahl 1982, 1994). In diesem Zusammenhang betont der Bürgermeister das Erfordernis offener Rechtfertigungskommunikation, welche den Entscheidungsfindungsprozess transparent und seine Ergebnisse nachvollziehbar machen kann:

teilweise liegen die auch diametral auseinander, die Wünsche (...) Und alles ist auch nicht finanzierbar und rechtlich machbar, was da geäußert worden ist. Also da wird noch mal so ein Punkt kommen, wo ich hoffe einfach, dass Menschen auch verstehen, dass das eben nicht geht. Und dann auch eine Entscheidung hinnehmen. (Interview 09)

Analog dazu wurde mit Blick auf Online-Beteiligung die Relevanz von EchtzeitInformationen zum Status quo, z. B. zum aktuellen Bearbeitungsstand, betont - ,eine Statusanzeige“ darüber, ob (und warum) die jeweilige Idee weiterverfolgt oder abgewiesen wird, stellt demnach einen zentralen Gelingensfaktor digitaler Beteiligungsprozessen dar. Hier zeigt sich ein entscheidender Ansatzpunkt für stärker responsive Online-Beteiligung: die Rückkopplung durch transparente, nachvollziehbare Entscheidungsschritte im Sinne permanenter Legitimationsketten. So kann besser plausibilisiert werden, dass sich ein konkretes Ergebnis in einem Prozess der sorgfältigen Abwägung und Integration zahlreicher, teils stark divergierender Eingaben und Interessenlagen herausbildet. Während solche Resultate im Regelfall mehreren Bedürfnissen entsprechen, finden zwangsläufig bestimmte Präferenzen weniger Berücksichtigung, was sich aber begründen und somit nachvollziehen lässt (Habermas 1992, 1999, 2011).

\subsection{Anforderungen an technische Lösungen für digitale Beteiligungsangebote}

Als zentraler Erfolgsfaktor für Online-Beteiligungsformate wird die Funktionalität (,moderierte Kommentarfunktion“, „Bildsprache nutzen“, „Interaktivität“) betont. Folgendes Zitat fasst die Kernanforderungen der Befragten gut zusammen: 
Sie [die App] ist visuell zu erfassen, intuitiv zu bedienen und sie wird moderiert. Das sind die drei Dinge, die ich für so eine Sache als unabdingbar empfinde. Und dass wirklich jeder, der einen Computer anmachen kann, sie auch bedienen kann, ohne großartig etwas machen zu müssen. Natürlich, das Ganze auch auf Smartphone und Tablets ... (Interview 11)

Von Seiten der interviewten KommunalpolitikerInnen wurde zudem die Einfachheit der Nutzung als weitere zentrale Anforderung angeführt. Die befragten Jugendlichen nutzen - u. a. aufgrund der Visualisierungen und Möglichkeit zur Vorsortierung - besonders gern Instagram, was die Bedeutung einer ansprechenden und verständlichen Aufbereitung unterstreicht:

Also ich denke Instagram ist so eine Plattform, wo man mehr Dinge visualisieren kann und das dann auch einfacher zu verstehen ist für die meisten Leute. (...) Also wenn man jetzt zum Beispiel ... hier wie mit dem Bürgerforum ... Könnte man einfach so ein Bild machen mit mehreren Menschen und dann schreibt man da was darunter. (...) Dann sieht man halt direkt: Ah okay. Da sind viele Leute. Da geht's um 'ne Versammlung. (Interview 32)

In nahezu jedem Interview finden Visualisierungs-, Diskussions-, Abstimmungsund Informationsfunktionen Erwähnung. Unter Visualisierungsfunktionen werden überwiegend interaktive Karten verstanden, die Bürger-Vorschläge zu Standorten kommunaler Einrichtungen und Infrastrukturen erlauben. Zudem wurden Visualisierungsmöglichkeiten zur Veranschaulichung geplanter Bauvorhaben angeregt, um eine bessere Entscheidungsgrundlage bereitzustellen (was bspw. durch Augmented Reality erreicht werden kann). Der Einsatz von Civic Tech und Online-Visualisierung gestaltet sich indes nicht immer einfach - so wird in einem Interview berichtet, dass viele BürgerInnen in einer Visualisierung bereits einen fest gesetzten Entwurf vermuteten, weshalb die Beteiligungsoption expliziert werden musste:

Ok, man möchte das natürlich mal irgendwo visualisiert haben und und und, aber damit war für viele Leute der Eindruck entstanden: Hm, wenn das alles schon so konkret geplant ist, was wollen wir jetzt dabei? Jetzt haben sie das Plakat ausgetauscht, da steht jetzt drauf: Sie haben jetzt wirklich die Möglichkeit, mitzugestalten. (Interview 13)

Im Rahmen der Bürger-Inputs für das zuvor erwähnte ISEK wurden einerseits übergeordnete und abstrakte Anliegen vorgebracht. Andererseits wurden konkrete Vorschläge gemacht - etwa Ideen bezüglich gewünschter architektonischer Veränderungen. Daraus lassen sich zwei wesentliche Anforderungen an das OnlineBeteiligungssystem ableiten: Zum einen soll das System den BenutzerInnen die Möglichkeit geben, ihre Eingaben abstrakt und in Textform auszudrücken. Zum anderen braucht es die Option, konkrete Ideen zu visualisieren. Diskussions- und Vorschlagsfunktionen gelten allgemein als Bereicherung, sofern es gelingt, die Erwartungen der Teilnehmenden realistisch zu halten sowie Hate Speech und Spam zu vermeiden. Hier werden mehrheitlich eine hohe Transparenz des Verfahrens sowie passende Moderationslösungen gefordert. Die Möglichkeit, Input (hinsichtlich Themen und Vorschlägen) ergebnisoffen beizutragen, wird tendenziell positiver be- 
wertet als geschlossene Verfahren. In diesem Zusammenhang wurde auch häufiger der Wunsch nach einem individualisierbaren System geäußert, das es ermöglicht, Verfahren gezielt an ein konkretes Vorhaben anzupassen und verschiedene Funktionen bedarfsgerecht zusammenzustellen. Die Jugendlichen gaben an, die geplante App sollte nicht zu viel Speicherplatz verbrauchen. Hier ließe sich gegebenenfalls auf eine browserbasierte Applikation zurückgreifen, zumal die Nutzbarkeit nicht nur über das Smartphone gegeben sein sollte. Schließlich sollte das Design ansprechend sein und in rudimentärem Umfang an den Anwendungskontext (z. B. die Kommune oder Region) angepasst werden können (Header, Titel und Schriftbild, ggf. Hintergrundfoto), um die Identifikation mit dem Angebot zu erhöhen.

\section{Fazit}

\subsection{Zusammenfassung zentraler Ergebnisse}

Wie sich zeigt, birgt die onlinebasierte Einbindung von BürgerInnen in Entscheidungsfindungsprozesse erhebliche Potenziale für die Stadt- und Regionalentwicklung. Sowohl die Reichweite als auch die Qualität der Informierung können erhöht und ein entsprechendes Interesse bei der Bevölkerung konnte nachgewiesen werden. KommunalpolitikerInnen stehen den Optionen positiv gegenüber und streben mehrheitlich an, Verbindungen zwischen BürgerInnen, Politik und Verwaltung zu verbessern. Sowohl mobile Applikationen als auch erweiterte interaktive Angebote wie Visualisierungstechniken, Ideenwettbewerbe, kreative Aufarbeitung von Informationen sowie gebündelte Stellungnahmen von Politik und Verwaltung bieten die Möglichkeit, über die üblichen Informierungs- und Dialogformate hinauszukommen. Während sich ausgewählter Input jeweils in das andere Format einspeisen lässt, ist bislang unklar, wie analoge und internetgestützte Beteiligungsangebote unmittelbarer und enger verzahnt werden können.

Ein weiteres zentrales Ergebnis besteht in der besonderen Bedeutung von Kontextfaktoren (Förster und Kaukal 2016; Gabriel und Kersting 2014; Rowe und Frewer 2005). So zeigten unmittelbare Rückmeldungen in den Workshops, dass die Attraktivität von Beteiligungsprozessen gesteigert werden kann, wenn entsprechend spezialisierte, orts- bzw. themenbezogene Angebote inklusive kreativen Gestaltungsoptionen zur Verfügung gestellt werden. Dies bestätigt die Bedeutung am unmittelbaren Beteiligungskontext ausgerichteter Applikationen und Instrumente (Korn 2013).

\subsection{Diskussion und Ausblick}

Aus den empirischen Erkenntnissen folgen zwei wesentliche Herausforderungen für gelingende Beteiligung: sie erfordert individuelle Ressourcen und ein verständiges Management zur Abstimmung digitaler und analoger Formate (Kim und Lee 2012). Zahlreiche Kommunen sind hiermit finanziell und personell sowie bezogen auf erforderliche Kompetenzen überfordert. Eine permanente Begleitung von Beteiligungsverfahren als auch die stetige Rückkopplung mit Prozessen (der Entscheidungsfindung, Implementierung, Evaluation etc.) stellen Ansprüche dar, denen 
realiter kaum entsprochen werden kann. Daher wirkt eine pragmatische Orientierung in diesem Zusammenhang angemessen (Steenbergen 2015). Was lässt sich nun - basierend auf den Erkenntnissen - zur Eignung von Online-Beteiligungsformaten, die Legitimität und Responsivität politischer Entscheidungen auf kommunaler Ebene zu erhöhen, festhalten? Anknüpfend an die drei zuvor entwickelten Kriterien Interessenallokation, Anschlussfähigkeit und Rückkopplung/Feedback, können die zusätzlichen Optionen einen besseren Anschluss von BürgerInnen-Präferenzen an Kommunalpolitik und Verwaltung gewährleisten; die Feedback-Optionen vermögen immerhin einen Mindeststandard zu garantieren. Gerade bei der Interessenallokation bestehen jedoch erhebliche Herausforderungen, die im Wesentlichen auf die (oftmals gleichzeitig zu leistende)

- Einbindung der BürgerInnen über einen längeren Zeitraum,

- Schaffung von Anknüpfungspunkten zwischen informellem Input und formellen Entscheidungsfindungsprozessen,

- (rasche) Verarbeitung großer Datenmengen (Auswertung und Bündelung),

- Interessenaggregation im engeren Sinne (Abbildung und Reduktion von Heterogenität und Komplexität) und

- die Erwartung von raschen Rückmeldungen zum aktuellen Stand des Projekts und zum Umgang mit eingebrachten Vorschlägen

zurückgeführt werden können. Weiterhin würde, selbst wenn all diese Maßgaben erfüllt werden könnten, das Hauptproblem der digital gespaltenen Demokratie (Sunstein 2017) bestehen bleiben: Für de facto Wenige werden immer mehr Input-Optionen bereitgestellt, während die Mehrheit der Betroffenen unberücksichtigt bleibt. Im Ergebnis kann dies die Distanz zwischen verschiedenen Bevölkerungsgruppen sowie zwischen der Bevölkerung und öffentlichen Institutionen vergrößern. Wir plädieren daher nicht für die Einführung weiterer (digitaler) Beteiligungsinstrumente, sondern für eine Integration multipler Optionen auf der einen Seite, sowie eine stärkere Rückbindung an politische Prozesse auf der anderen Seite (wenngleich aufgrund von Sachzwängen nur eine Auswahl an Eingaben berücksichtigt, eine begrenzte Anzahl an Feedback-Schleifen durchgeführt und damit stets nur Ergebnisse erzielt werden können, die demokratie- und beteiligungstheoretischen Idealen nur begrenzt genügen). Es bestehen zudem Potentiale für eine Optimierung der Angebotsstrukturen und Nutzungsraten durch gezielte Anreize. Im vorliegenden Fall zeigt sich, dass es zum einen Spielräume für einen kreativ-offenen Input-Prozess braucht (konkret: relativ frei beplanbare Flächen) und zum anderen aus Sicht der zu Beteiligenden spezifische Ansatzpunkte identifiziert und für relevant befunden werden müssen, um sie zur Teilnahme zu motivieren. Überlegungen zur stärkeren Einbindung von BürgerInnen, etwa durch Übertragung bestimmter Aufgaben (zum Beispiel die Pflegeund Moderation von Online-Plattformen), rekurrieren auf Selbstverantwortung und Eigenständigkeit als höchste Stufe der Beteiligung (Arnstein 1969) und sind grundsätzlich begrüßenswert, wenngleich in der Folge ein sensibles und aufwendigeres Management notwendig ist, um die Einhaltung von Standards zu gewährleisten. Weiterhin ist der Nutzen lokaler Instrumente zeitlich begrenzt; immerhin bestehen oft Übertragbarkeiten auf andere Kontexte. Der Adaptierbarkeit kommt also besondere Bedeutung zu: Unter den genannten Bedingungen ist es gerade für öffentliche 
Vorhabenträger von Vorteil, die kontextübergreifende Anwendbarkeit von Formaten zu fördern. Tatsächlich arbeiten diverse Bundesländer an Online-Portalen, die einschlägige Angebote für die Öffentlichkeitsbeteiligung bereitstellen. Allerdings weisen die meisten darin gesammelten Instrumente geringe Kontextsensibilität und Innovativität auf.

Abschließend kann konstatiert werden, dass Online-Beteiligung keineswegs bekannte Schwächen kollektiver Willensbildungsprozessen transzendiert, jedoch durchaus Angebote bereitstellen kann, deren Wert sui generis unabhängig von anderen Faktoren besteht. In der Literatur wird jenseits einer partizipativen oder direkten Demokratie eine Stärkung der Repräsentation, ein besseres Zusammenwirken von BürgerInnen und etablierter Politik vorgeschlagen (Schüttemeyer 2020). Die Idee einer stärker input-orientierten Demokratie durch Repräsentationsbeziehungen (Hadamitzky 2016) kann durch Online-Beteiligung befördert werden, indem die Nähe zu lokalen Institutionen erhöht wird. Zugegeben: die empirische Forschung zeigt, dass dies bislang nicht in erheblichem Maße geschieht und die beteiligten BürgerInnen sich überwiegend aus jenen rekrutieren, die bereits in anderen Kontexten aktiv und engagiert sind. Indes geht die Abwendung von der Demokratie häufig mit größerer Distanz zu lokaler Politik einher (Agerberg 2017). Wir bewerten daher die Nähe als einen wesentlichen Faktor für vitale, repräsentativ verfasste Demokratien. Die gegenwärtig verstärkte Nutzung digitaler Formate zeigt: Zahlreiche Optionen können erschlossen werden, um Distanzen zu überbrücken, wobei auch die Grenzen des Digitalen aufscheinen, da die physische Präsenz mitunter unabdingbar scheint. Digitale Formate können den realweltlichen demokratischen Austausch daher nicht ersetzen, aber sie stellen wertvolle Ergänzungen dar, indem sie die Agora potentiell für jedermann erweitern und öffnen.

Danksagung Wir danken in besonderem Maße Marco Becker, Nino Bohn, Emily Drewing, David Schlabach, Sebastian Schwarzloh und Timur Sereflioglu für die Unterstützung bei der Erstellung dieses Beitrages.

Förderung Die empirische Erhebung ist Teil des Forschungsprojektes „Creactive Citizen“, das mit Mitteln des Europäischen Fonds für regionale Entwicklung (EFRE) gefördert wird.

Funding Open Access funding provided by Projekt DEAL.

Open Access Dieser Artikel wird unter der Creative Commons Namensnennung 4.0 International Lizenz veröffentlicht, welche die Nutzung, Vervielfältigung, Bearbeitung, Verbreitung und Wiedergabe in jeglichem Medium und Format erlaubt, sofern Sie den/die ursprünglichen Autor(en) und die Quelle ordnungsgemäß nennen, einen Link zur Creative Commons Lizenz beifügen und angeben, ob Änderungen vorgenommen wurden.

Die in diesem Artikel enthaltenen Bilder und sonstiges Drittmaterial unterliegen ebenfalls der genannten Creative Commons Lizenz, sofern sich aus der Abbildungslegende nichts anderes ergibt. Sofern das betreffende Material nicht unter der genannten Creative Commons Lizenz steht und die betreffende Handlung nicht nach gesetzlichen Vorschriften erlaubt ist, ist für die oben aufgeführten Weiterverwendungen des Materials die Einwilligung des jeweiligen Rechteinhabers einzuholen.

Weitere Details zur Lizenz entnehmen Sie bitte der Lizenzinformation auf http://creativecommons.org/ licenses/by/4.0/deed.de. 


\section{Literatur}

Aragonès, Enriqueta, und Santiago Sánchez-Pagés. 2009. A theory of participatory democracy based on the real case of Porto Alegre. European Economic Review 53(1):56-72.

Åström, Joachim, Mikael Granberg, und Abdul Khakee. 2011. Apple pie-spinach metaphor: shall e-democracy make participatory planning more wholesome? Planning Practice \& Research 26(5):571-586.

Abdu, Shamsu Dauda, Bahtiar Mohamad, und Suhaini Muda. 2017. Youth Online political participation: the role of Facebook use, Interactivity, quality information and political interest. In SHS Web of Conferences 33: 00080, Hrsg. B. Mohamad, H. Abu Bakar https://doi.org/10.1051/shsconf/20173300080.

van Ackern, Gertrud. 2017. Political participation in local politics. The case of Tübingen. In Local politics in a comparative perspective, Hrsg. Rolf Frankenberger, Elena Chernenkova, 151-162. BadenBaden: Nomos.

Agerberg, Mattias. 2017. Failed expectations: Quality of government and support for populist parties in Europe. European Journal of Political Research 56(3):578-600.

Alcaide Muñoz, Laura, Francisco José, und Alcaraz Quiles. 2018. Optimizing E-participation initiatives through social media. Hershey: IGI Global. https://doi.org/10.4018/978-1-5225-5326-7.

Alcántara, Sophia, Rainer Kuhn, Ortwin Renn, Nicolas Bach, Birgit Böhm, Hans-Liudger Dienel, Peter Ullrich, Carolin Schröder, und Heike Walk. 2014. DELIKAT - Fachdialoge Deliberative Demokratie: Analyse Partizipativer Verfahren für den Transformationsprozess. Dessau: Umweltbundesamt. https://www.umweltbundesamt.de/sites/default/files/medien/378/publikationen/texte_31_2014_ delikat-fachdialoge_deliberative_demokratie.pdf. Zugegriffen: 20. Mai 2020.

Alonso, Ángel Iglesias. 2009. E-Participation And Local Governance: A Case Study. Theoretical and Empirical Researches in Urban Management 4(3(12)):49-62.

Anter, Andreas. 2019. Die Krise der Repräsentation. Warum erfolgreiche populistische Parteien auf Schwächen im repräsentativen System verweisen. In Repräsentation, Hrsg. Rüdiger Voigt, 239-252. BadenBaden: Nomos.

Arnstein, Sherry R. 1969. A ladder of citizen participation. Journal of the American Institute of Planners 35(4):216-224.

Auschner, Eika. 2008. Kommunaler Bürgerhaushalt: Das brasilianische Erfolgsmodell und dessen Umsetzung in Deutschland. Saarbrücken: VDM.

Baack, Stefan, Christian Djeffal, Juliane Jarke, und Hendrik Send. 2020. Civic Tech. In Handbuch Digitalisierung in Staat und Verwaltung, Hrsg. Tanja Klenk, Frank Nullmeier, und Göttrik Wewer, 1-9. Wiesbaden: Springer VS. https://doi.org/10.1007/978-3-658-23669-4_29-1.

Bartonitz, Martin, Veronika Lévesque, Thomas Michl, Wolf Steinbrecher, Cornelia Vonhof, und Ludger Wagner (Hrsg.). 2018. Agile Verwaltung: Wie der Öffentliche Dienst aus der Gegenwart die Zukunft entwickeln kann. Berlin, Heidelberg: Gabler.

Bieber, Christoph. 2018. Partizipation in der Piratenpartei. Die Schattenseiten einer sonnigen Utopie. Politische Vierteljahresschrift 59(2):403-404.

Bizjak, Igor. 2012. Improving public participation in spatial planning with web 2.0 tools. Urbani izziv 23(1):112-124.

Blühdorn, Ingolfur. 2013. Simulative Demokratie. Neue Politik nach der postdemokratischen Wende. Berlin: Suhrkamp.

Booher, David E. 2018. Planning with complexity: an introduction to collaborative rationality for public policy, 2. Aufl., London: Routledge.

Boulianne, Shelley. 2009. Does internet use affect engagement? A meta-analysis of research. Political Communication 26:193-211.

Boulianne, Shelley. 2018. Twenty years of digital media effects on civic and political participation. Communication Research https://doi.org/10.1177/0093650218808186.

Brady, Henry E., Sidney Verba, und Kay Lehman Schlozman. 1995. Beyond Ses: a resource model of political participation. The American Political Science Review 89(2):271-294.

Brettschneider, Frank (Hrsg.). 2020. Bau- und Infrastrukturprojekte: Dialogorientierte Kommunikation als Erfolgsfaktor. Wiesbaden: Springer VS.

Bude, Heinz, und Philipp Staab (Hrsg.). 2016. Kapitalismus und Ungleichheit: Die neuen Verwerfungen. Frankfurt a.M.: Campus.

Butterwegge, Christoph. 2020. Die zerrissene Republik: Wirtschaftliche, soziale und politische Ungleichheit in Deutschland, 2. Aufl., Weinheim: Beltz.

Campbell, Angus, Philip Converse, Warren Miller, und Donald Stokes. 1960. The American Voter. New York: Wiley. 
Charest, Francine, Johanie Bouffard, und Ema Zajmovic. 2016. Public relations and social media: Deliberate or creative strategic planning. Public Relations Review 42(4):530-538.

Collin, Philippa. 2015. Young citizens and political participation in a digital society: addressing the democratic disconnect. London: Palgrave Macmillan.

Collingridge, David. 1980. The social control of technology. London: Pinter.

Conroy, Meredith, und Justin Vaughn. 2018. Undermining the message: how social media can sabotage strategic political communication actions. In Strategische Politische Kommunikation im digitalen Wandel, Hrsg. Michael Oswald, Michael Johann, 97-113. Wiesbaden: Springer VS.

Conroy, Maria Manta, und Jennifer Evans-Cowley. 2006. E-participation in planning: an analysis of cities adopting on-line citizen participation tools. Environment and Planning C: Government and Policy 24(3):371-384.

Dahl, Robert Alan. 1982. Dilemmas of pluralist democracy: autonomy vs. Control. New Haven: Yale University Press.

Dahl, Robert Alan. 1994. A democratic dilemma: system effectiveness versus citizen participation. Political Science Quarterly 109(1):23-34.

Damurski, Lukasz. 2012. E-participation in urban planning: Online tools for citizen engagement in Poland and in Germany. International Journal of E-Planning Research 1(3):40-67.

van Dijk, Jan A.G.M. 2006. Digital Divide Research, Achievements and Shortcomings. Poetics 34(4):221-235.

Dolde, Klaus-Peter. 2013. Neue Formen der Bürgerbeteiligung? Planung und Zulassung von Projekten in der parlamentarischen Demokratie. Neue Zeitschrift für Verwaltungsrecht 32(12):769-775.

Döllner, Jürgen, Thomas Kolbe, Falko Liecke, Takis Sgouros, und Karin Teichmann. 2006. The virtual 3D city model of Berlin-Managing, integrating, and communicating complex urban information. Proceedings of the 25th Urban Data Management Symposium UDMS. https://mediatum.ub.tum.de/ doc/1145759/1145759.pdf. Zugegriffen: 20. Mai 2020.

Donders, Maud, Thomas Hartmann, und Anita Kokx. 2014. E-participation in urban planning: getting and keeping citizens involved. International Journal of E-Planning Research 3(2):54-69.

Dong, Chuqing, und Yingru Ji. 2018. Connecting young adults to democracy via government social network sites. Public Relations Review 44(5):762-775.

Easton, David. 1965. A systems analysis of political life. New York: Wiley.

Eckardt, Frank. 2016. Urban Governance und „e-Participation“? Innovative Politik in der medialisierten Stadt. In Inclusive City, Hrsg. Melanie Behrens, Wolf-Dietrich Bukow, Karin Cudak, und Christoph Strünck, 161-172. Wiesbaden: Springer VS.

Eisel, Stephan. 2016. Technikfaszination braucht Demokratiekompetenz: Zur Jugendbeteiligung im Internet. In Politische Beteiligung junger Menschen, Hrsg. Jörg Tremmel, Markus Rutsche, 271-294. Wiesbaden: Springer VS.

Eom, Seok-Jin, Hanchan Hwang, und Kim Jun Houng. 2018. Can social media increase government responsiveness? A case study of Seoul, Korea. Government Information Quarterly 35(1):109-122.

Ertelt, Jürgen. 2012. Mehr Beteiligung realisieren durch digitale Medien und Internet: ePartizipation schafft gestaltende Zugänge für Jugendliche zur Demokratieentwicklung. Sozial Extra: Zeitschrift Für soziale Arbeit 36(9):47-50.

Escher, Tobias. 2013. Does the use of the Internet further democratic participation? A comparison of citizens' interactions with political representatives in the UK and Germany. Oxford: Oxford University Research Archive.

Escher, Tobias, und Rosar Ulrich. 2016. Der Einfluss von Online-Verfahren auf die Legitimität demokratischer Entscheidungen. Zeitschrift für Vergleichende Politikwissenschaft 10(2):179-211.

Escher, Tobias, und Rottinghaus Bastian. 2019. The Impact of ICTs on Public Support for Democracy: Evidence from a Comparative Case Study of Online Consultations in Three German Municipalities. Vortrag DVPW-Konferenz „Governance of Big Transformations“ am 21. März 2019, München: HfP. http://www.bigtransformations.hfp.tum.de/wp-content/uploads/2019/12/TagungsberichtGovernance-of-Big-Transformations-München-2019final.pdf. Zugegriffen: 20. Mai 2020.

Eulau, Heinz, und Paul D. Karps. 1977. The puzzle of representation: specifying components of responsiveness. Legislative Studies Quarterly 2:233-254.

Feeney, Mary K., und Welch Eric. 2012. Electronic participation technologies and perceived outcomes for local government managers. Public Management Review 14(6):815-833.

Flügge, Erik, und Lucas Gerrits. 2016. Kontinuierliche Beteiligung und viele Beteiligte zugleich - ein unlösbarer Widerspruch? In Politische Beteiligung junger Menschen: Grundlagen - Perspektiven Fallstudien, Hrsg. Jörg Tremmel, Markus Rutsche, 413-434. Wiesbaden: Springer VS. 
Forrester, Maya, und Matusitz Jonathan. 2010. A narrowing digital divide: the impact of the Internet on youth political participation. Communicare: Journal for Communication Sciences in Southern Africa 29(2):85-98.

Förster, André, und Malte Kaukal. 2016. Unkonventionelle politische Partizipation in Deutschland: Haben Kontextfaktoren auf Kreisebene einen Einfluss? Politische Vierteljahresschrift 57(3):353-377.

Franzke, Jochen, und Heinz Kleger. 2009. Bürgerhaushalt ohne Bürger? Analyse der Ergebnisse einer Einwohnerbefragung in der Stadt Potsdam im Frühjahr 2007. Potsdam: Universitätsverlag.

Gabriel, Oscar W., und Norbert Kersting. 2014. Politisches Engagement in deutschen Kommunen: Strukturen und Wirkungen auf die politischen Einstellungen von Bürgerschaft, Politik und Verwaltung. In Partizipation im Wandel. Unsere Demokratie zwischen Wählen, Mitmachen und Entscheiden, Hrsg. Bertelsmann-Stiftung, Staatsministerium Baden-Württemberg, 43-181. Gütersloh: Bertelsmann Stiftung.

Galston, William A., James Hunter Davison, und John M. Owen. 2018. Anti-pluralism: the populist threat to liberal democracy. New Haven: Yale University Press.

Garcia, Ana Cristina Bicharra, Fernando B. Pinto, und Inhauma Neves Ferraz. 2005. Electronic participatory budgeting (e-PPB): increasing people participation in the decision-making process. International Journal of Web Based Communities 1 (4):504

Gauld, Robin, Jayde Flett, Sasha McComb, Andrew Gray. 2016. How responsive are government agencies when contacted by email? Findings from a longitudinal study in Australia and New Zealand. Government Information Quarterly 33 (2):283-290

Geis, Anna, Frank Nullmeier, und Christopher Daase (Hrsg.). 2012. Der Aufstieg der Legitimitätspolitik: Rechtfertigung und Kritik politisch-ökonomischer Ordnungen. Baden-Baden: Nomos.

Gibson, Rachel, und Marta Cantijoch. 2013. Conceptualizing and Measuring Participation in the Age of the Internet: Is Online Political Engagement Really Different to Offline? The Journal of Politics 75(3):701-716.

Giebler, Heiko. 2020. Gute Entscheidungen in der Demokratie aus Bevölkerungs- und Elitensicht. In Legitimitätsprobleme: Zur Lage der Demokratie in Deutschland, Hrsg. Sascha Kneip, Wolfgang Merkel, und Bernhard Weßels, 223-252. Wiesbaden: Springer VS.

Gilman, Hollie Russon. 2017. Civic tech for urban collaborative governance. PS: Political Science \& Politics 50(3):744-750.

Gilman, und Hollie Russon. 2016. Democracy reinvented. Participatory budgeting and civic innovation in America. Washington, DC: Brookings Institution.

Große, Katharina. 2018. Benutzerzentrierte E-Partizipation: Typologie, Anforderungen und Gestaltungsempfehlungen. Wiesbaden: Springer VS.

de Haan, Gerhard. 2008. Gestaltungskompetenz als Kompetenzkonzept der Bildung für nachhaltige Entwicklung. In Kompetenzen der Bildung für nachhaltige Entwicklung: Operationalisierung, Messung, Rahmenbedingungen, Befunde, Hrsg. Inka Bormann, Gerhard de Haan, 23-43. Wiesbaden: Springer VS.

Habermas, Jürgen. 1992. Drei normative Modelle der Demokratie: zum Begriff deliberativer Politik. In Die Chancen der Freiheit: Grundprobleme der Demokratie, Hrsg. Iring Fetscher, Herfried Münkler, 11-24. München: Piper.

Habermas, Jürgen. 1999. Wahrheit und Rechtfertigung: philosophische Aufsätze. Frankfurt am Main: Suhrkamp.

Habermas, Jürgen. 2011. Theorie des kommunikativen Handelns. Frankfurt am Main: Suhrkamp.

Hadamitzky, Sebastian. 2016. Demokratische Qualität in Deutschland. Baden-Baden: Nomos.

Head, Brian W. 2011. Why not ask them? Mapping and promoting youth participation. Children and Youth Services Review 33(4):541-547.

Healey, Patsy. 2003. Collaborative planning in perspective. Planning Theory 2(2):101-123.

Healey, Patsy. 2006. Collaborative planning: shaping places in fragmented societies, 2. Aufl., Basingstoke: Palgrave Macmillan.

Hennemann, Max, und Claus C. Wiegandt. 2010. E-Partizipation in der Stadtplanung: Chancen und Grenzen der Bürgerbeteiligung im Informationszeitalter. In E-Government und Stadtentwicklung, Hrsg. Lena Hatzelhoffer, Michael Lobeck, Wolfgang Müller, und Claus C. Wiegandt, 99-113. Münster: LIT.

Hill, Hermann, Mario Martini, und Edgar Wagner (Hrsg.). 2014. Transparenz, Partizipation, Kollaboration: die digitale Verwaltung neu denken. Baden-Baden: Nomos.

Hoffmann, Christian Pieter, Jasmin Weber, Robert Zepic, Vanessa Greger, und Helmut Krcmar. 2019. Dimensionen digitaler Mündigkeit und politische Beteiligung im Netz. In Politische Partizipation 
im Medienwandel, Hrsg. Ines Engelmann, Marie Legrand, und Hanna Marzinkowski, 79-99. Berlin: Böhland \& Schremmer.

Holtkamp, Lars. 2008. Bürgerhaushalt. In Politische Beteiligung. Einführung in dialogorientierte Instrumente politischer und gesellschaftlicher Partizipation, Hrsg. Norbert Kersting, 222-235. Wiesbaden: Springer VS.

Hordijk, Michaela. 2005. Participatory governance in Peru: exercising citizenship. Environment and Urbanization 17 (1):219-236

Hugger, Kai-Uwe, und Markus Walber (Hrsg.). 2010. Digitale Lernwelten. Wiesbaden: Springer VS.

IFOK GmbH. 2018. Bürgergutachten 2030: BAYERN, DEINE ZUKUNFT. München: Bayerische Staatskanzlei. https://www.bayern.de/wp-content//uploads/2017/06/bayern-2030-buergergutachtengesamt_final.pdf. Zugegriffen: 20. Mai 2020.

Innes, Judith E., und David E. Booher. 2003. The Impact of Collaborative Planning on Governance Capacity. Berkeley: University of California. https://escholarship.org/uc/item/98k72547. Zugegriffen: 15. Mai 2019.

Innes, Judith E., und David E. Booher. 2010. Planning with Complexity: An Introduction to Collaborative Rationality for Public Policy. New York: Routledge.

Jobst, Markus, Jürgen Döllner, und Olaf Lubanski. 2010. Communicating Geoinformation effectively with virtual 3d city models. In Handbook of research on E-planning: ICTs for urban development and monitoring, Hrsg. Carlos Nunes Silva, 120-142. Hershey: IGI Global.

Johanning, Anja. 2009. Kompetenzentwicklung im Internet. Baden-Baden: Nomos.

Jörke, Dirk. 2019. Die Größe der Demokratie: Über die räumliche Dimension von Herrschaft und Partizipation. Berlin: Suhrkamp.

Jost, Pablo, Marc Ziegele, und Teresa K. Naab. 2020. Klicken oder tippen? Eine Analyse verschiedener Interventionsstrategien in unzivilen Online-Diskussionen auf Facebook. Zeitschrift für Politikwissenschaft https://doi.org/10.1007/s41358-020-00212-9.

Kallus, Rachel. 2016. Citizenship in action: participatory urban visualization in contested urban space. Journal of Urban Design 21(5):616-637.

Kanhere, Salil S. 2013. Participatory sensing: crowdsourcing data from mobile Smartphones in Urban spaces. In Distributed computing and Internet technology, lecture notes in computer science, Hrsg. Chittaranjan Hota, Pradip K. Srimani, 19-26. Berlin, Heidelberg: Springer.

Kearns, Ian, Jamie Bend, und Beatrice Stern. 2002. E-Participation in Local Government. London: Institute for Public Policy Research.

Kelly, Eric Damian. 2010. Community planning: an introduction to the comprehensive plan, 2. Aufl., Washington, DC: Island Press.

Kersting, Norbert. 2016. Online- und Offlinebeteiligung als lokale demokratische Innovation - Die Sicht der Ratsmitglieder. In Politik mit Bürgern - Politik für Bürger: Praxis und Perspektiven einer neuen Beteiligungskultur, Hrsg. Manuela Glaab, 91-110. Wiesbaden: Springer VS.

Kersting, Norbert. 2019. Online Partizipation: Evaluation und Entwicklung - Status quo und Zukunft. In Politik in der digitalen Gesellschaft, Hrsg. Jeanette Hofmann, Norbert Kersting, Claudia Ritzi, und Wolf J. Schünemann, 105-122. Bielefeld: transcript.

Kersting, Norbert, und Phillip Hocks. 2019. Politische Online- und Offline-Partizipation junger Menschen. In Handbuch Philosophie der Kindheit, Hrsg. Johannes Drerup, Gottfried Schweiger, 413-418. Stuttgart: J.B. Metzler.

Kim, Soonhee, und Jooho Lee. 2012. E-participation, transparency, and trust in local government. Public Administration Review 72:819-828.

Kim, Soojin, und Hindy Lauer Schachter. 2013. Citizen participation in the budget process and local government accountability. Case studies of organizational learning from the United States and South Korea. Public Performance \& Management Review 36(3):456-471.

Klages, Helmut. 2013. Bürgerbeteiligung auf kommunaler Ebene. Baden-Baden: Nomos.

Klages, Helmut, und Carmen Daramus. 2007. Bürgerhaushalt Berlin-Lichtenberg. Partizipative Haushaltsplanaufstellung, -entscheidung und -kontrolle im Bezirk Lichtenberg von Berlin. Speyer: Deutsches Forschungsinstitut für öffentliche Verwaltung.

Klammer, Julia, Fred Van den Anker, und Markus Nollert. 2017. E-Partizipation in der Stadtentwicklung: Kritische Erfolgsfaktoren, Mehrwert und Anforderungen an die Umsetzung. In Emanzipation, Soziale Arbeit und Stadtentwicklung. Eine programmatische und methodische Herausforderung, Hrsg. Patrick Oehler, Nadine Käser, Matthias Drilling, Jutta Guhl, und Nicola Thomas, 339-362. Berlin, Toronto: Budrich. 
Kneip, Sascha, und Wolfgang Merkel. 2020. Demokratische Legitimität: Ein theoretisches Konzept in empirisch-analytischer Absicht. In Legitimitätsprobleme: Zur Lage der Demokratie in Deutschland, Hrsg. Sascha Kneip, Wolfgang Merkel, und Bernhard Weßels, 25-55. Wiesbaden: Springer VS.

Korn, Matthias. 2013. Situating engagement: ubiquitous infrastructures for in-situ civic engagement. Aarhus: Aarhus University.

Kubicek, Herbert. 2010. The potential of E-participation in urban planning: a European perspective. In Handbook of research on E-planning: ICTs for Urban development and monitoring, Hrsg. Carlos Nunes Silva, 168-194. Hershey: IGI Global.

Kubicek, Herbert. 2014. Staatliche Beteiligungsangebote im Internet - Ein Überblick. In Internet und Partizipation, Hrsg. Kathrin Voss, 263-298. Wiesbaden: Springer VS.

Kubicek, Herbert. 2017. E-Partizipation: Welchen Unterschied macht das „E“? Informatik-Spektrum 40:270-277.

Kumar, Ashok, und Ronan Paddison. 2000. Trust and collaborative planning theory: the case of the scottish planning system. International Planning Studies 5(2):205-223.

Leffers, Donald. 2019. Communicative planning. In The Wiley Blackwell encyclopedia of urban and regional studies, Hrsg. Anthony M. Orum, 1-3. Hoboken: Wiley-Blackwell. https://doi.org/10.1002/ 9781118568446.eurs0055.

Linden, Markus, und Winfried Thaa (Hrsg.). 2014. Ungleichheit und politische Repräsentation. BadenBaden: Nomos.

Loomans, Dirk. 2015. Gefahren digitaler Politikvermittlung - Zwischen Shopping Mall und Basar. In Digitale Politikvermittlung: Chancen und Risiken interaktiver Medien, Hrsg. Mike Friedrichsen, Roland A. Kohn, 427-439. Wiesbaden: Springer VS.

Lupia, Arthur, und Mathew D. McCubbins. 1998. The democratic dilemma: Can citizens learn what they need to know? Cambridge: Cambridge University Press.

Macintosh, Ann. 2004. Characterizing e-participation in policy-making. In 37th Annual Hawaii International Conference on System Sciences https://doi.org/10.1109/HICSS.2004.1265300.

Macintosh, Ann, Edmund Robson, Ella Smith, und Angus Whyte. 2003. Electronic democracy and young people. Social Science Computer Review 21(1):43-54.

Macintosh, Ann, Thomas F. Gordon, Alastair Renton. 2009. Providing Argument Support for E-Participation. Journal of Information Technology \& Politics 6 (1):43-59

Margerum, Richard D. 2002. Collaborative planning: building consensus and building a distinct model for practice. Journal of Planning Education and Research 21(3):237-253.

Marr, Mirko, und Nicole Zillien. 2019. Digitale Spaltung. In Handbuch Online-Kommunikation, Hrsg. Wolfgang Schweiger, Klaus Beck, 283-306. Wiesbaden: Springer VS.

Masser, Kai, Adriano Pistoia, und Philipp Nitzsche (Hrsg.). 2013. Bürgerbeteiligung und Web 2.0: Potentiale und Risiken webgestützter Bürgerhaushalte. Wiesbaden: Springer VS.

May, Andrew, und Tracy Ross. 2018. The design of civic technology: factors that influence public participation and impact. Ergonomics 61(2):214-225.

McNulty, Stephanie L. 2015. Barriers to participation: exploring gender in Peru's participatory budget process. Journal of Development Studies 51(11):1429-1443.

Meckel, Miriam, Christan P. Hoffmann, Christoph Lutz, und Robin Poëll. 2014. DIVSI-Studie zu Bereichen und Formen der Beteiligung im Internet: Ein Überblick über den Stand der Forschung. Hamburg, St. Gallen: Deutsches Institut für Vertrauen und Sicherheit im Internet (DIVSI), Institut für Medien- und Kommunikationsmanagement.

Meinel, Florian. 2019. Vertrauensfrage: Zur Krise des heutigen Parlamentarismus. München: C.H.Beck.

Merkel, Wolfgang, und Claudia Ritzi. 2017. Die Legitimität direkter Demokratie. Wie demokratisch sind Volksabstimmungen? Wiesbaden: Springer VS.

Michels, Ank. 2011. Innovations in democratic governance: how does citizen participation contribute to a better democracy? International Review of Administrative Sciences 77:275-293.

Michels, Ank. 2012. Citizen participation in local policy making: design and democracy. International Journal of Public Administration 35(4):285-292.

Michels, Ank, und Laurens De Graaf. 2017. Examining citizen participation: local participatory policymaking and democracy revisited. Local Government Studies 43(6):875-881.

Miller, Warren E., und Donald E. Stokes. 1963. Constituency influence in congress. American Political Science Review 57:45-56.

Modica, Giuseppe, Paolo Zoccali, und Salvatore Di Fazio. 2013. The e-participation in tranquillity areas identification as a key factor for sustainable landscape planning. In Computational science and its applications-ICCSA 2013, Hrsg. Beniamino Murgante, Sanjay Misra, Maurizio Carlini, Carmelo 
M. Torre, Hong-Quang Nguyen, David Taniar, Bernady O. Apduhan, und Osvaldo Gervasi, 550-565. Berlin, Heidelberg: Springer.

Moser, Sonja. 2010. Beteiligt sein: Partizipation aus der Sicht von Jugendlichen. Wiesbaden: Springer VS.

Mossberger, Karen, Yonghong Wu, und Jared Crawford. 2013. Connecting citizens and local governments? Social media and interactivity in major U.S. cities. Government Information Quarterly 30(4):351-358.

Muncke, Günter. 1972. Zur Demokratisierung des kommunalpolitischen Planungs- und Entscheidungsprozesses. Zeitschrift für Parlamentsfragen 3(2):230-244.

Nam, Taewoo. 2012. Suggesting frameworks of citizen-sourcing via Government 2.0. Government Information Quarterly 29(1):12-20.

Naranjo Zolotov, Mijail, Tiago Oliveira, und Sven Casteleyn. 2018. E-participation adoption models research in the last 17 years: A weight and meta-analytical review. Computers in Human Behavior $81: 350-365$.

Niederberger, Marlen, und Stefanie Dreiack. 2018. Inklusive Teilnahme zwischen Fiktion und Realität? Eine Diskussion des Potenzials von On- und Offline-Partizipation am Beispiel des Bürgerdialogs Zukunftsthemen. In Strategische Politische Kommunikation im digitalen Wandel: Interdisziplinäre Perspektiven auf ein dynamisches Forschungsfeld, Hrsg. Michael Oswald, Michael Johann, 259-288. Wiesbaden: Springer VS.

Norris, Pippa. 2012. Political mobilization and social networks.: The example of the Arab spring. In Electronic Democracy, Hrsg. Norbert Kersting, Michael Stein, und John Trent, 55-76. Opladen: Barbara Budrich.

Oser, Jennifer, Marc Hooghe, und Sofie Marien. 2013. Is Online participation distinct from offline participation? A latent class analysis of participation types and their stratification. Political Research Quarterly 66(1):91-101.

Pitkin, Hanna F. 1967. The concept of representation. Berkeley: University of California Press.

Pollino, Maurizio, und Giuseppe Modica. 2013. Free web mapping tools to characterise landscape dynamics and to favour e-participation. In Computational science and its applications-ICCSA 2013, Hrsg. Beniamino Murgante, Sanjay Misra, Maurizio Carlini, Carmelo M. Torre, Hong-Quang Nguyen, David Taniar, Bernady O. Apduhan, und Osvaldo Gervasi, 566-581. Berlin, Heidelberg: Springer.

Rademacher, Lars, Klaus Lintemeier, und Heiko Kretschmer. 2020. Öffentlichkeitsbeteiligung bei Infrastrukturprojekten als Herausforderung für Politik und Verwaltung. In Öffentliche Verwaltung - Verwaltung in der Öffentlichkeit: Herausforderungen und Chancen der Kommunikation öffentlicher Institutionen, Hrsg. Klaus Kocks, Susanne Knorre, und Jan Niklas Kocks, 165-184. Wiesbaden: Springer VS.

Reddick, Christopher, und Donald F. Norris. 2013. E-participation in local governments: an examination of political-managerial support and impacts. Transforming Government: People, Process and Policy 7(4):453-476.

Reddick, Christopher G., Akemi Takeoka Chatfield, und Adegboyega Ojo. 2017. A social media text analytics framework for double-loop learning for citizen-centric public services: A case study of a local government Facebook use. Government Information Quarterly 34:110-125.

Rehbein, Boike, Benjamin Baumann, Luzia Costa, und Simin Fadaee (Hrsg.). 2015. Reproduktion sozialer Ungleichheit in Deutschland. Konstanz, München: UVK.

Robra-Bissantz, Susanne, Felix Becker, Pascal Abel, und Cedric Handke. 2017. Das ,e-“ in Partizipation. HMD Praxis der Wirtschaftsinformatik 54(4):461-476.

Rölle, Daniel. 2020. Agile Verwaltung. In Handbuch Digitalisierung in Staat und Verwaltung, Hrsg. Tanja Klenk, Frank Nullmeier, und Göttrik Wewer, 1-11. Wiesbaden: Springer VS. https://doi.org/10.1007/ 978-3-658-23669-4_12-1.

Römmele, Andrea, und Henrik Schober. 2013. The governance of large-scale projects: linking citizens and the state. Baden-Baden: Nomos.

Rosanvallon, Pierre. 2011. Democratic legitimacy impartiality, reflexivity, proximity. Princeton: Princeton University Press.

Rossmann, Doralyn, und Elizabeth A. Shanahan. 2012. Defining and achieving normative democratic values in participatory budgeting processes. Public Administration Review 72(1):56-66.

Rotondo, Francesco. 2012. The U-city paradigm: opportunities and risks for E-democracy in collaborative planning. Future Internet 4(2):563-574.

Rowe, Gene, und Lynn J. Frewer. 2005. A typology of public engagement mechanisms. Science, Technology \& Human Values 30(2):251-290.

Rudolph, Steffen. 2019. Digitale Medien, Partizipation und Ungleichheit: Eine Studie zum sozialen Gebrauch des Internets. Wiesbaden: Springer VS. 
Runciman, David. 2020. So endet die Demokratie. Frankfurt a.M.: Campus.

Saad-Sulonen, Joanna. 2012. The role of the creation and sharing of digital media content in participatory E-planning. International Journal of E-Planning Research 1(2):1-22. https://doi.org/10.4018/ijepr. 2012040101.

Sarcinelli, Ulrich. 2013. Kommunikation und Partizipation in einer veränderten Legitimationsarchitektur. In Das Internet: Bereicherung oder Stressfaktor für die Demokratie?, Hrsg. Marianne Kneuer, 104-122. Baden-Baden: Nomos.

Saridar Masri, Sawsan. 2018. Integrating youth in city planning: developing a participatory tool toward a child-friendly vision of Eastern Wastani - Saida. Alexandria Engineering Journal 57(2):897-909.

Scherer, Sabrina, und Maria A. Wimmer. 2011. Reference framework for E-participation projects. In Electronic participation, Hrsg. Efthimios Tambouris, Ann Macintosh, und Hans de Bruijn, 145-156. Berlin, Heidelberg: Springer.

Schlozman, Kay, und Henry Brady. 2018. Unequal and unrepresented: political inequality and the people's voice in the new gilded Age. Princeton: Princeton University Press.

Schlozman Lehman, Kay, Sidney Verba, und Henry E. Brady. 2010. Weapon of the strong? Participatory inequality and the Internet. Perspectives on Politics 8(02):487-509.

Schmidthuber, Lisa, Dennis Hilgers, Thomas Gegenhuber, und Stefan Etzelstorfer. 2017. The emergence of local open government: determinants of citizen participation in online service reporting. Government Information Quarterly 34(3):457-469.

Schrotter, Gerhard, und Christian Hürzeler. 2020. The digital twin of the city of Zurich for urban planning. PFG-Journal of Photogrammetry, Remote Sensing and Geoinformation Science 88(1):99-112.

Schüttemeyer, Suzanne S. 2020. Repräsentative Demokratie und politische Partizipation. In Partizipation für alle und alles? Fallstricke, Grenzen und Möglichkeiten, Hrsg. Astrid Lorenz, Christian Pieter Hoffmann, und Uwe Hitschfeld, 183-202. Wiesbaden: Springer VS.

Shu, Hang, und Xiaoqing Gu. 2018. Determining the differences between online and face-to-face student-group interactions in a blended learning course. The Internet and Higher Education 39:13-21.

Silva, Carlos Nunes. 2010. Handbook of research on e-planning: ICTs for urban development and monitoring. Hershey: IGI Global.

Soßdorf, Anna. 2016. Zwischen Like-Button und Parteibuch: die Rolle des Internets in der politischen Partizipation Jugendlicher. Wiesbaden: Springer VS.

Steenbergen, Marco R. 2015. The Democratic Dilemma Revisited. In Politische Psychologie, Hrsg. Thorsten Faas, Cornelia Frank, und Harald Schoen, 492-510. Baden-Baden: Nomos.

Streeck, Wolfgang. 2013. Gekaufte Zeit: Die vertagte Krise des demokratischen Kapitalismus. Berlin: Suhrkamp.

Sunstein, Cass R. 2017. \#Republic: divided democracy in the Age of social media. Oxford: Princeton University Press.

Szyszka, Peter. 2020. Die Krux öffentlicher Verwaltungskommunikation - Public Relations der öffentlichen Verwaltung. In Öffentliche Verwaltung - Verwaltung in der Öffentlichkeit: Herausforderungen und Chancen der Kommunikation öffentlicher Institutionen, Hrsg. Klaus Kocks, Susanne Knorre, und Jan Niklas Kocks, 13-37. Wiesbaden: Springer VS.

Sæbø, Øystein, Leif Skiftenes Flak, und Maung K. Sein. 2011. Understanding the dynamics in e-Participation initiatives: looking through the genre and stakeholder lenses. Government Information Quarterly 28:416-425.

Taube, Alex, Xiaomao Wu, und Ganesh Pingale. 2020. GritWorld 3D-Real-Time-Digital-Smart-City-Plattform. In Smart City - Made in Germany: Die Smart-City-Bewegung als Treiber einer gesellschaftlichen Transformation, Hrsg. Chirine Etezadzadeh, 647-657. Wiesbaden: Springer VS.

Thiele, Alexander. 2019. Legitimität in unsicheren Zeiten: Der demokratische Verfassungsstaat in der Krise? Tübingen: Mohr Siebeck.

Träger, Hendrik. 2020. Die Legitimität politischer Beteiligungsformate im Lichte von Zugangsvoraussetzungen und Nutzungsanforderungen. In Partizipation für alle und alles? Fallstricke, Grenzen und Möglichkeiten, Hrsg. Astrid Lorenz, Christian Pieter Hoffmann, und Uwe Hitschfeld, 221-240. Wiesbaden: Springer VS.

Vanslambrouck, Silke, Chang Zhu, Koen Lombaerts, Brent Philipsen, und Jo Tondeur. 2018. Students' motivation and subjective task value of participating in online and blended learning environments. The Internet and Higher Education 36:33-40.

Vickery, Jacqueline Ryan. 2017. Worried About the Wrong Things: Youth, Risk, and Opportunity in the Digital World. Cambridge, MA: The MIT Press. 
Weiß, Jens. 2018. Evaluation von Projekten zur Förderung der politischen Partizipation Jugendlicher auf Kreisebene. In Perspektiven der angewandten Verwaltungsforschung in Deutschland, Hrsg. Joachim Beck, Jürgen Stember, 211-230. Baden-Baden: Nomos.

Wesselmann, Christoph. 2002. Internet und Partizipation in Kommunen: Strategien des optimalen Kommunikations-Mix. Wiesbaden: Dt. Univ.-Verl.

Wiegandt, Claus-Christian, Michael Lobeck, Oliver Märker, Konstantin Wolf, und Johannes Häußler. 2018. Webbasierte Medien in der Stadtentwicklung: Bürgerbeteiligung und Bürgerengagement in der digitalen Gesellschaft. Bonn: Bundesinstitut für Bau-, Stadt- und Raumforschung.

Wilker, Nadja. 2019. Online-Bürgerbeteiligung und politische Repräsentation. Rollen und Einstellungen kommunaler Mandatsträger in der digitalen Demokratie. Wiesbaden: Springer VS.

Wilson, James Lindley. 2019. Democratic Equality. Princeton: Princeton University Press.

Wintermantel, Vanessa. 2020. Der Wert der Vielfalt: Gesellschaftliche Pluralität, Meinungsvielfalt und demokratische Legitimität. In Legitimitätsprobleme: Zur Lage der Demokratie in Deutschland, Hrsg. Sascha Kneip, Wolfgang Merkel, und Bernhard Weßels, 255-286. Wiesbaden: Springer VS.

Zhang, Sisi, und Antoni B. Moore. 2014. The usability of online geographic virtual reality for urban planning. In Innovations in 3D Geo-information sciences, Hrsg. Umit Isikdag, 225-242. Cham: Springer.

Zheng, Yueping. 2017. Explaining citizens' E-participation usage: functionality of E-participation applications. Administration \& Society 49(3):423-442.

Zittel, Thomas. 2010. Mehr Responsivität durch neue digitale Medien? Baden-Baden: Nomos.

Zobel, Maria-Stephanie, und Jens Weiß. 2018. Wirkung von E-Partizipation: Erkenntnisse aus Evaluationen elektronischer Bürgerhaushalte. In Wirkungen von E-Government: Impulse für eine wirkungsgesteuerte und technikinduzierte Verwaltungsreform, Hrsg. Jürgen Stember, Wolfgang Eixelsberger, und Andreas Spichiger, 207-245. Wiesbaden: Springer VS. 